\title{
DETERMINATION OF 24 PESTICIDES RESIDUES IN LEATHER PRODUCTS BY SOLID-PHASE MICROEXTRACTION COUPLED WITH GAS CHROMATOGRAPHY-MASS SPECTROMETRY
}

\author{
Jinlan DAI ${ }^{1,2,3}$, Honglei YIN³ , Hang WEI ${ }^{4}$, Lei ZHOU ${ }^{5}$, Minghua LIU6* \\ ${ }^{1}$ College of Chemical Engineering, Fuzhou University, Fuzhou, 350116, Fujian Province, China; 29171239@qq.com \\ ${ }^{2}$ Technical Center of Fuzhou Customs District of P.R.China, 350003, China \\ ${ }^{3}$ Fujian Key Laboratory for Technology Research of Inspection and Quarantine, 350003, China; 21558049@qq.com \\ ${ }^{4}$ Institute of Quality Standards \& Testing Technology for Agro-products, Fujian Academy of Agricultural Sciences/Fujian Key \\ Laboratory of Agro-products Quality and Safety, Fuzhou 350003, China; 349537891@qq.com \\ ${ }^{5}$ Technical Center for Safety of Industrial Products of Tianjin Customs District of P .R.China, 300308, China; \\ zhouleiciq@126.com \\ ${ }^{6}$ College of Environment and Resource, Fuzhou University, Fuzhou 350116, Fujian Province, China; mhliu2000@fzu.edu.cn
}

Received: 09.09.2020

Accepted: 26.11 .2020

https://doi.org/10.24264/lfj.20.4.5

DETERMINATION OF 24 PESTICIDES RESIDUES IN LEATHER PRODUCTS BY SOLID-PHASE MICROEXTRACTION COUPLED WITH GAS CHROMATOGRAPHY-MASS SPECTROMETRY

ABSTRACT. Considering the high content of oil and complex residual additives in leather samples, a new analytical method based on the solidphase extraction technique and gas chromatography-selected ion monitoring mass spectrometry (GC-SIM-MS) was developed to determine 24 organic compounds involving the organochlorine pesticides (OCPs), organophosphorous pesticides (OPPs) and pyrethroids pesticides residues in leather. The extraction conditions (such as the extraction solution, purification procedure and solid-phase extraction column) were optimized using the positive leather samples based on the recovery rates of the pesticides. The best extraction solution, solid-phase extraction column and chromatography column were $n$-hexane and ethyl acetate $(1+1$, volume) mixed solution, Carb-PSA (1.0 g, $6 \mathrm{~mL})$ and DB-1701 (length: $30 \mathrm{~m}$, inside diameter: $0.25 \mathrm{~mm}$, film thickness: $0.25 \mu \mathrm{m}$ ). The optimized extraction time and temperature were $20 \mathrm{~min}$ and $25^{\circ} \mathrm{C}$, respectively. The detection limits of 24 pesticide residues range from 0.05 to $0.10 \mathrm{mg} / \mathrm{kg}$, and the recoveries range from $74 \%$ to $116 \%$. The relative standard deviations (RSD, $n=6$ ) range from $5.42 \%$ to $12.00 \%$. The developed method presented a simple, rapid, sensitive, and inexpensive method to detect 24 pesticides in skin and leather and was successfully applied to the detect them in leather products (cowhide, sheep leather and pig leather).

KEY WORDS: leather, organochlorine pesticides, organophosphorous pesticides, pyrethroids pesticides, GC-MS

DETERMINAREA A 24 DE REZIDUURI DE PESTICIDE ÎN PRODUSELE DIN PIELE PRIN MICROEXTRACȚIE ÎN FAZĂ SOLIDĂ CUPLATĂ CU CROMATOGRAFIE DE GAZE - SPECTROMETRIE DE MASĂ

REZUMAT. Având în vedere conținutul ridicat de ulei și aditivi reziduali complecși din probele de piele, s-a dezvoltat o nouă metodă analitică bazată pe tehnica de extracție în fază solidă și cromatografie de gaze cuplată cu spectrometria de masă, prin monitorizarea ionilor selecționați (GC-SIM-MS) pentru a determina reziduurile a 24 de compuși organici printre care pesticide organoclorurate (OCP), pesticide organofosforice (OPP) și piretroizi. Condițiile de extracție (cum ar fi soluția de extracție, procedura de purificare și coloana de extracție în fază solidă) au fost optimizate folosind probe de piele pozitive pe baza ratelor de recuperare a pesticidelor. Cea mai bună soluție de extracție, coloană de extracție în fază solidă și coloană de cromatografie au fost soluția mixtă de n-hexan și acetat de etil (1+1, volum), Carb-PSA (1,0 g, 6 ml) și DB-1701 (lungime: $30 \mathrm{~m}$, diametru interior: 0,25 mm, grosime film: 0,25 $\mu \mathrm{m}$ ). Timpul și temperatura de extracție optimizate au fost de $20 \mathrm{~min}$ și respectiv $25^{\circ} \mathrm{C}$. Limitele de detecție a celor 24 de reziduuri de pesticide variază de la $0,05 \mathrm{la} 0,10 \mathrm{mg} / \mathrm{kg}$, iar recuperările variază de la $74 \%$ la 116\%. Abaterile standard relative (RSD, $n=6$ ) variază de la 5,42\% la 12,00\%. Metoda dezvoltată pentru a detecta 24 de pesticide în piele este simplă, rapidă, sensibilă și ieftină, și a fost aplicată cu succes pentru a detecta aceste reziduuri în produse din piele (de vacă, de oaie și de porc).

CUVINTE CHEIE: piele, pesticide organoclorurate, pesticide organofosforice, pesticide piretroide, GC-MS

\begin{abstract}
DÉTERMINATION DE 24 RÉSIDUS DE PESTICIDES DANS LES PRODUITS EN CUIR PAR MICROEXTRACTION EN PHASE SOLIDE COUPLÉE AVEC LA CHROMATOGRAPHIE GAZEUSE - SPECTROMÉTRIE DE MASSE

RÉSUMÉ. Compte tenu de la teneur élevée en huile et en additifs résiduels complexes dans les échantillons de cuir, une nouvelle méthode analytique basée sur la technique d'extraction en phase solide et la chromatographie en phase gazeuse couplée avec la spectrométrie de masse à contrôle d'ions sélectionnés (GC-SIM-MS) a été développée pour déterminer 24 composés organiques impliquant les pesticides organochlorés (OCP), les pesticides organophosphorés (OPP) et les pesticides pyréthrinoïdes dans le cuir. Les conditions d'extraction (telles que la solution d'extraction, la procédure de purification et la colonne d'extraction en phase solide) ont été optimisées en utilisant les échantillons de cuir positifs basés sur les taux de récupération des pesticides. La meilleure solution d'extraction, la colonne d'extraction en phase solide et la colonne de chromatographie étaient une solution mixte de $\mathrm{n}$-hexane et d'acétate d'éthyle ( $1+1$, volume), Carb-PSA ( $1,0 \mathrm{~g}$, $6 \mathrm{ml}$ ) et DB-1701 (longueur : $30 \mathrm{~m}$, diamètre à l'intérieur : 0,25 mm, épaisseur du film : 0,25 $\mu \mathrm{m}$ ). Le temps et la température d'extraction optimisés étaient respectivement de $20 \mathrm{~min}$ et $25^{\circ} \mathrm{C}$. Les limites de détection de 24 résidus de pesticides vont de 0,05 à $0,10 \mathrm{mg} / \mathrm{kg}$ et les taux de récupération vont de $74 \%$ à $116 \%$. Les écarts types relatifs ( $R S D, n=6)$ vont de $5,42 \%$ à $12,00 \%$. La méthode développée pour détecter 24 pesticides dans la peau est une méthode simple, rapide, sensible et peu coûteuse et a été appliquée avec succès pour les détecter les résidus dans les produits en cuir (vache, mouton et porc).

MOTS CLÉS : cuir, pesticides organochlorés, pesticides organophosphorés, pesticides pyréthrinoïdes, GC-MS
\end{abstract}

* Correspondence to: Minghua LIU, College of Environment and Resource, Fuzhou University, Fuzhou 350116, Fujian Province, China; mhliu2000@fzu.edu.cn 


\section{INTRODUCTION}

Skins and leather were widely used in clothing, shoes, automobile, packaging, and decoration industry [1-3]. To obtain good quality of leather, a variety of pesticides were often used in the different stage of leather storage and production [4, 5]. In 1950s, when the raw skin was preserved by air drying in Africa, DDT and lindane were used widely to protect raw skin from putrefaction. Organochlorine and dieldrin were applied to protect against ectoparasites. Lindane was widely used to protect hides and skins from insects in 1990s [6,7].

Pesticide is a substance or mix-up substances which were used to prevent, destroy, repel, or lessen the damage of any injurious insects. According to the source of raw materials, there are many kinds of pesticide, such as chemical substance, biological agent (such as virus or bacteria), antimicrobial or disinfectant. Some of the used pesticides are persistent organic pollutants (POPs). Compared to conventional pollutants, POPs can cause more hazards on human health and the natural environment due to their persistence, bioaccumulation and high toxicity [8, 9]. The persistence of these compounds in the environment is due to their low degradation by biotic and abiotic process, leading to a long half-live time [8]. Because most of the pesticides in leather, especially OCPs are lipophilic, can be absorbed by living organisms through alimentation, breathing and the skin. After absorption, these compounds usually distributed in various tissue including blood [10-12]. The toxicity of these contaminants is very complex and is specific to each compound. Therefore, multiple toxic responses can occur according to the species, gender and organ affected [1315]. Previous reports showed that high levels of DDT and pentachlorophenol were detected in the children and adolescents of Germany, because of a leather jacket impregnated with some pesticides, pyrethroid, organophosphorus insecticides, pentachlorophenol (PCP), lindane $(\gamma-\mathrm{HCH})$, and dichloro(diphenyl)ethylene (DDE) $[16,17]$.

The trace pesticides in leather may come from livestock breeding process, delivery in the animal food chain, used as preservatives in storage, transportation or processing [18]. These additives may remain in the final products as volatile and semi-volatile organic compounds [8].

The critical substances potentially presented in footwear and footwear components are listed in ISO/TR 16178:2012 [19]. But there are very few sensitive analytical methodologies published and only few related standards and methods for testing pesticides residue in textiles [20-23]. Until now, there has been no responding fast detection method and standard for many kinds of pesticides involved in shoe materials, especially in leather. To protect environment and human health [24-27], it is urgent and necessary to develop methods and international detection standard to monitor and detect these substances.

This paper described a method for the quantitative analysis of 24 pesticide residues in leather. Using small sample volumes, solid phase extraction combined with GC-MS was used to identify 24 pesticide residues at trace concentrations.

\section{MATERIALS AND METHODS}

\section{Chemicals and Reagents}

Certified standards of the 24 kinds of pesticide (purity of $97 \%$ or more) were obtained from Dr.E of Germany. Their retention time, quantitative and qualitative ions and ion abundance ratios were shown in Table 1 . The standard stock solution of pesticides was $1 \mu \mathrm{g} /$ $\mathrm{ml}$ in $\mathrm{n}$-hexane. The standard stock solution was stored at $4^{\circ} \mathrm{C}$, and the shelf life is one month. The standard calibration solutions of pesticides are in the range of $10 \mathrm{ng} / \mathrm{ml}$ to $1000 \mathrm{ng} / \mathrm{ml}$, and were prepared before using. Chromatographic pure grade of $\mathrm{N}$-hexane, ethyl acetate, acetonitrile, toluene were purchased from TEDIA USA. Solid phase extraction (SPE) column is graphitized carbon black - ethylenediamine - N - propyl methyl silane (Carb - PSA, $1.0 \mathrm{~g}, 6 \mathrm{~mL}$ ), Carb-NH2, Carb-PSA, PSA, Carb-NH2, Florisil and Alumina $\mathrm{N}$. All the analytical solvents were supplied by Sigma-Aldrich. 
Table 1: Retention time, quantitative and qualitative ions and ion abundance ratios of the 24 pesticides

\begin{tabular}{|c|c|c|c|c|c|}
\hline \multirow{2}{*}{$\begin{array}{l}\text { Series } \\
\text { Number }\end{array}$} & \multirow[b]{2}{*}{ Pesticides } & \multirow{2}{*}{$\begin{array}{l}\text { Retention } \\
\text { time /min }\end{array}$} & \multicolumn{3}{|c|}{ Characteristic fragment ions /amu } \\
\hline & & & $\begin{array}{l}\text { Quantitative } \\
\text { ion }\end{array}$ & $\begin{array}{l}\text { Qualitative } \\
\text { ion }\end{array}$ & $\begin{array}{c}\text { Abundance } \\
\text { ratio }\end{array}$ \\
\hline 1 & Pentachloroanisole & 10.52 & 280 & $265,237,263$ & $100: 100: 82: 63$ \\
\hline 2 & $\alpha-B H C$ & 11.26 & 181 & $183,217,254$ & 100:97:69:4 \\
\hline 3 & Lindane & 12.41 & 181 & $183,217,254$ & 100:98:64:11 \\
\hline 4 & Aldrin & 13.78 & 263 & $265,261,293$ & $100: 67: 65: 38$ \\
\hline 5 & Chlorothalonil & 14.64 & 266 & 264,268 & $100: 78: 48$ \\
\hline 6 & $\beta-\mathrm{BHC}$ & 14.87 & 181 & $183,217,254$ & 100:98:74:9 \\
\hline 7 & $\delta-\mathrm{BHC}$ & 15.65 & 181 & $183,217,254$ & 100:97:70:8 \\
\hline 8 & Malathion & 15.84 & 173 & 158.256 .285 & $100: 48: 8: 5$ \\
\hline 9 & Dichlofluanide & 16.05 & 123 & $224,226,332$ & $100: 37: 26: 5$ \\
\hline 10 & Ethylparathion & 16.10 & 275 & $220,247,232$ & $100: 82: 77: 68$ \\
\hline 11 & Heptachloroepoxide & 16.36 & 353 & $355,351,317$ & $100: 81: 52: 68$ \\
\hline 12 & $o, p^{\prime}-D D E$ & 16.74 & 246 & $318,176,248$ & $100: 34: 29: 65$ \\
\hline 13 & $\alpha$-Endosulfan & 17.16 & 241 & $265,277,339$ & $100: 63: 57: 40$ \\
\hline 14 & Tolyfluanide & 17.85 & 238 & 240,181 & 100:69:63 \\
\hline 15 & $p, p^{\prime}-D D E$ & 17.97 & 318 & $316,246,248$ & 100:78:130:84 \\
\hline 16 & Dieldrin & 18.55 & 263 & $277,345,380$ & $100: 79: 27: 24$ \\
\hline 17 & $o, p^{\prime}-D D D$ & 19.21 & 235 & $237,165,199$ & $100: 65: 44: 17$ \\
\hline 18 & $o, p^{\prime}-D D T$ & 19.72 & 235 & $237,165,199$ & $100: 65: 40: 15$ \\
\hline 19 & $p, p^{\prime}-D D D$ & 21.10 & 235 & $237,165,199$ & $100: 64: 42: 12$ \\
\hline 20 & $\beta$-Endosulfan & 21.30 & 241 & $265,237,339$ & $100: 49: 84: 35$ \\
\hline 21 & $p, p^{\prime}-D D T$ & 21.68 & 235 & $237,165,199$ & 100:65:39:12 \\
\hline 22 & Mirex & 22.85 & 272 & $274,237,332$ & $100: 80: 53: 11$ \\
\hline 23 & Methoxychlor & 23.41 & 227 & $228,212,274$ & $100: 20: 17: 15$ \\
\hline \multirow{2}{*}{24} & & 25.70 & 183 & $163,165,184$ & $100: 20: 17: 15$ \\
\hline & Permethrin & 26.13 & 183 & $163,165,184$ & $100: 27: 22: 15$ \\
\hline
\end{tabular}

\section{Instrument}

SPE was performed using a Bond Elut Carbon column (Agilent technologies, USA). GCMS analysis was achieved using GC with massselective detector (MSD) and electron impact source (EIS). Separation was performed on Agilent DB-1701 capillary column $(30 \mathrm{~m} \times 0.25$ $\mathrm{mm}, 0.25 \mu \mathrm{m}$ film thickness). The optimized GCMS conditions were as follows: injection mode: without split; injector port temperature: $280^{\circ} \mathrm{C}$; injection volume: $1.0 \mu \mathrm{L}$; carrier gas: helium with flow rate $1.2 \mathrm{~mL} \mathrm{~min}^{-1}$. Oven program: $50^{\circ} \mathrm{C}$ for 2 min, increased to $185^{\circ} \mathrm{C}$ at $30.0^{\circ} \mathrm{C} \mathrm{min}^{-1}$, held for $1 \mathrm{~min}$ and raised to $240^{\circ} \mathrm{C}$ at a rate of $4.0^{\circ} \mathrm{C} \mathrm{min}^{-1}$ and raised to $270^{\circ} \mathrm{C}$ at a rate of $20.0^{\circ} \mathrm{C} \mathrm{min}^{-1}$ then held for $5 \mathrm{~min}$. Finally, selected $280^{\circ} \mathrm{C}$ and held for $2 \mathrm{~min}$ to remove impurities. All data were analyzed using Agilent Chem Station software.

\section{Methods}

Preparation of Positive Samples

The positive leather samples were prepared and just added the 24 pesticides to the samples in Jinjiang Chenxin Tannery according to usual process, meanwhile blank leather samples were prepared with the same process without pesticides.

Sample Extraction and Purification

Blank and leather samples $2.0 \mathrm{~g}$ was extracted with $20 \mathrm{~mL}$ hexane-ethyl acetate ( v/v 1:1), vortex oscillated for $10 \mathrm{~min}$ and then 
extracted for 20 min by ultrasonic extraction under $25^{\circ} \mathrm{C} \pm 2^{\circ} \mathrm{C}$. The extraction was centrifuged for $5 \mathrm{~min}$ at $5000 \mathrm{rpm}$, and the supernatant was transferred into a brown conical flask. The above procedure was repeated twice. Then combined supernatant was concentrated by a vacuum rotary evaporator at $35^{\circ} \mathrm{C} \pm 5^{\circ} \mathrm{C}$ to approximately $2 \mathrm{ml}$ to $5 \mathrm{ml}$ for further purification.

Before injection into the GC-MS system, the sample solution needs to be concentrated and purified. Firstly, SPE Carb column was prerinsed with acetonitrile- toluene $(3: 1, v / v)$, and the conical flask was rinsed twice with $3 \mathrm{~mL}$ acetonitrile and toluene $(3: 1, v / v)$ mixed solution and transfer the washing solutions to the Carb column.

Secondly, the extraction solution flew through the pre-prepared SPE Carb column, 15
$\mathrm{mL}$ acetonitrile-toluene $(3: 1, \mathrm{v} / \mathrm{v})$ as the eluent. Combined and concentrated the solution, then added $5 \mathrm{~mL}$ hexane to exchange the solvent and evaporated to be dry at $35^{\circ} \mathrm{C} \pm 5^{\circ} \mathrm{C}$ (Solvent displacement was used to change the polarity of the solvent to protect chromatographic column). Repeat this operation. Finally, $2.0 \mathrm{~mL}$ hexane was used to dissolve the analytes and the sample was filtered with $0.22 \mu \mathrm{m}$ membrane for further GC-MS analysis. The 100\% ion abundance of fragment ion in the mass spectrum of each underivatized drug was chosen as the quantitative ion. The selective representative ions of 24 kinds of pesticides for quantification were shown in Table 2.

Under the optimized instrument conditions, the chromatograms of 24 pesticides in the standard solutions were shown in Figure 1.

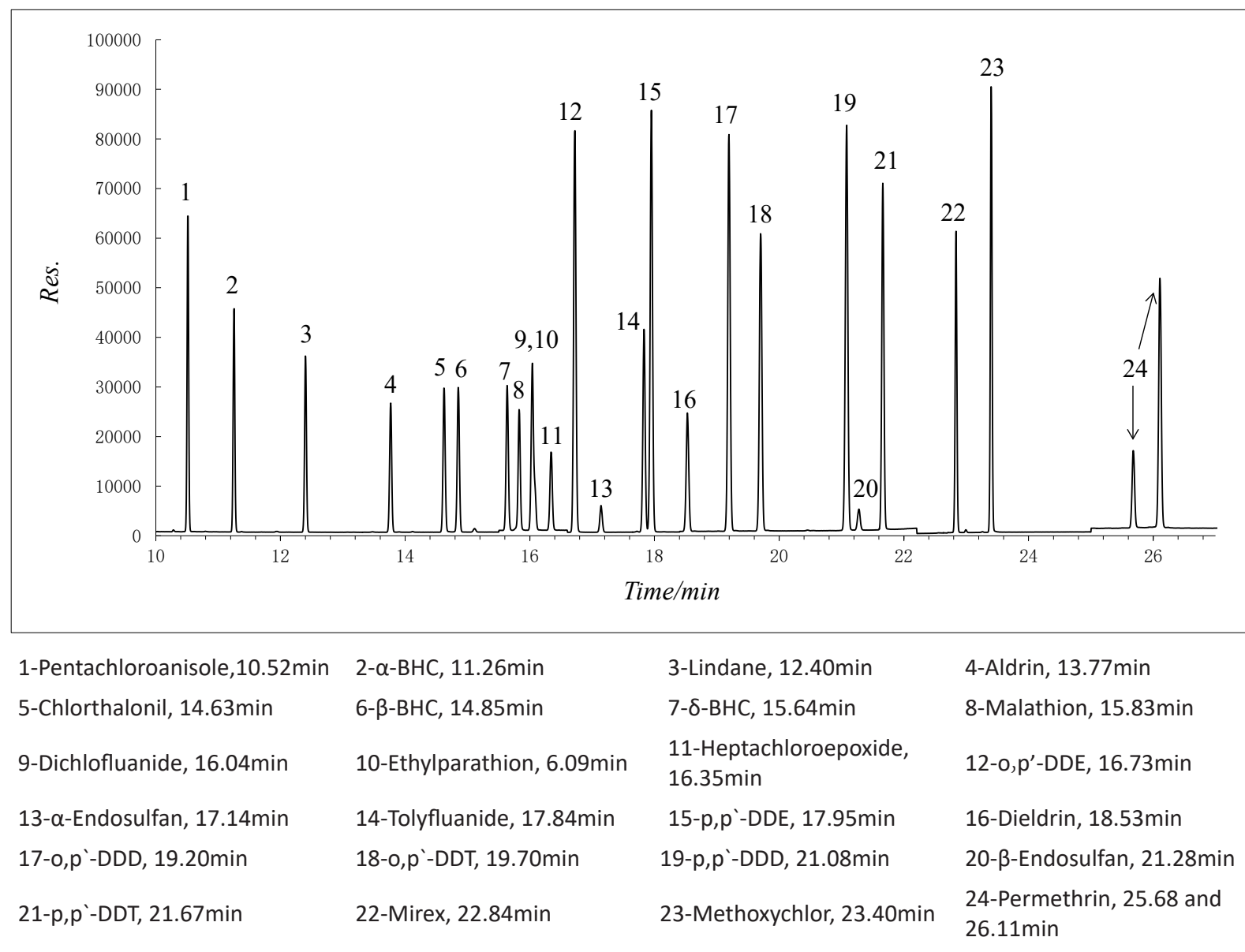

Figure 1. The chromatograms of 24 pesticides 


\section{RESULTS AND DISCUSSION}

\section{Method Development}

Through preliminary test, it was found out that hexane-ethyl acetate system was the suitable extraction solvent. So then the effect of extraction solvent on the efficiency was studied by different ratio of hexane and ethyl acetate $(5: 1,3: 1,2: 1,1: 1,1: 2,1: 3,1: 5, \mathrm{v} / \mathrm{v})$. The results showed that the recoveries of $p, p^{\prime}-D D D, p, p^{\prime}-$ DDT, methoxychlor were lower than $60 \%$ when the ratio was $5: 1,3: 1$, or $2: 1$. Besides, when the ratio was $1: 2,1: 3$, or $1: 5$, the recoveries of $p, p^{\prime}-$ DDD, p, p'-DDT, methoxychlor were 85-107\%, which satisfied the requirement, but those of chlorothalonil, dichlofluanide, tolyfluanide were very low. Generally considering the total analytes, the ratio 1:1 was chosen as the optimum ratio of hexane and ethyl acetate solvent system. The experimental results were shown in supplemental materials (Table S2).

To optimize the extraction efficiency, three extraction methods (votex oscillation, sonication, mixed votex and sonication) were evaluated. The experiments results showed that the extraction efficiency of the analytes was the highest when hexane-ethyl acetate $(1: 1, v / v)$ was used as the solvent and sonicated for $20 \mathrm{~min}$ after votex oscillation for $10 \mathrm{~min}$ at $25^{\circ} \mathrm{C}$.

The extraction efficiency at different extraction time was studied. The test samples were extracted for $10 \mathrm{~min}, 20 \mathrm{~min}, 30 \mathrm{~min}, 40$ $\mathrm{min}, 50 \mathrm{~min}$ and $60 \mathrm{~min}$ at the temperature of $25^{\circ} \mathrm{C} \pm 2{ }^{\circ} \mathrm{C}$. Each sample was tested twice, and the results are shown in Figure 2. From the results, it was found that with the extraction time increasing, the pesticide content increased in the beginning, and then decreased. For most of the 24 kinds of pesticide (except tolyfluanide and chlorothalonil), the concentration reached the highest when the extraction time was 20 min. So $20 \mathrm{~min}$ was chosen as the optimum extraction time.

To study the effect of the temperature on the extraction efficiency, the test samples were extracted at the temperatures of $25^{\circ} \mathrm{C}, 30^{\circ} \mathrm{C}, 35^{\circ} \mathrm{C}$, and $40^{\circ} \mathrm{C}$ for $20 \mathrm{~min}$, respectively. It was found that the highest extraction content was obtained at the temperature of $25^{\circ} \mathrm{C}$. So the temperature of $25^{\circ} \mathrm{C}$ was chosen as the optimized extraction temperature. The result for each test piece was the average content of the two-test piece, and the test results are shown in Figure 3.

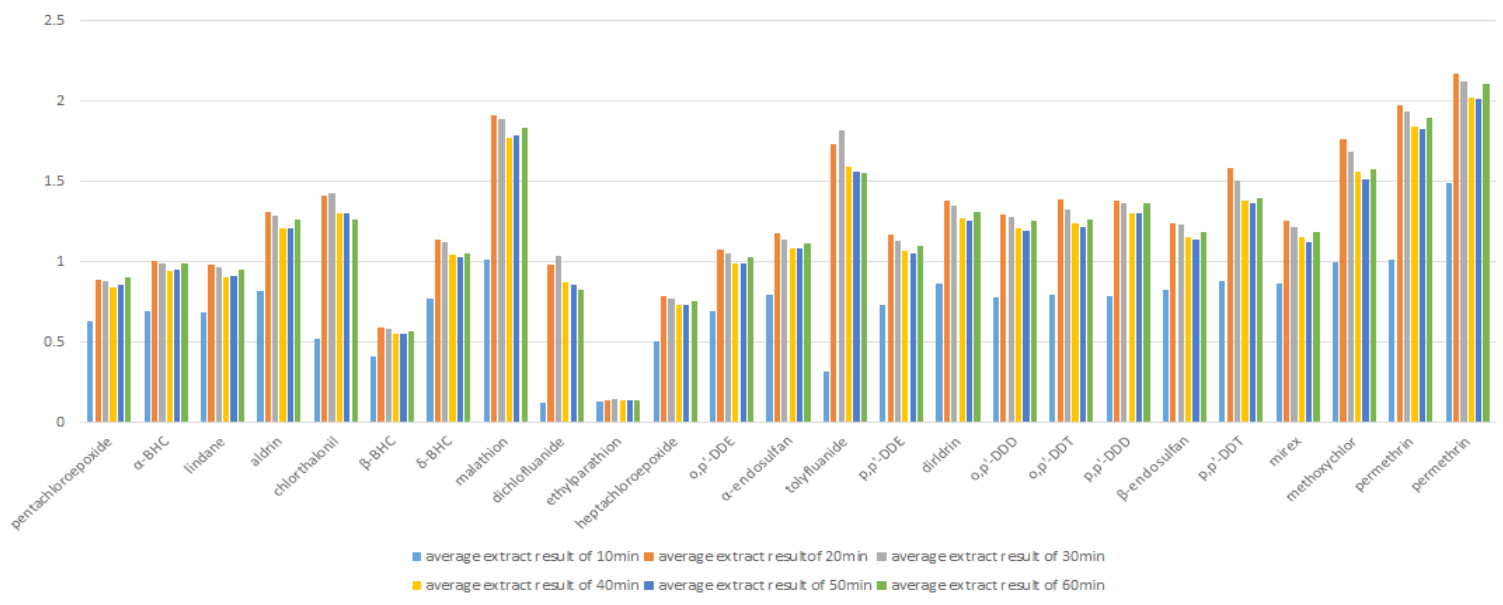

Figure 2. Extraction results at different extraction time 


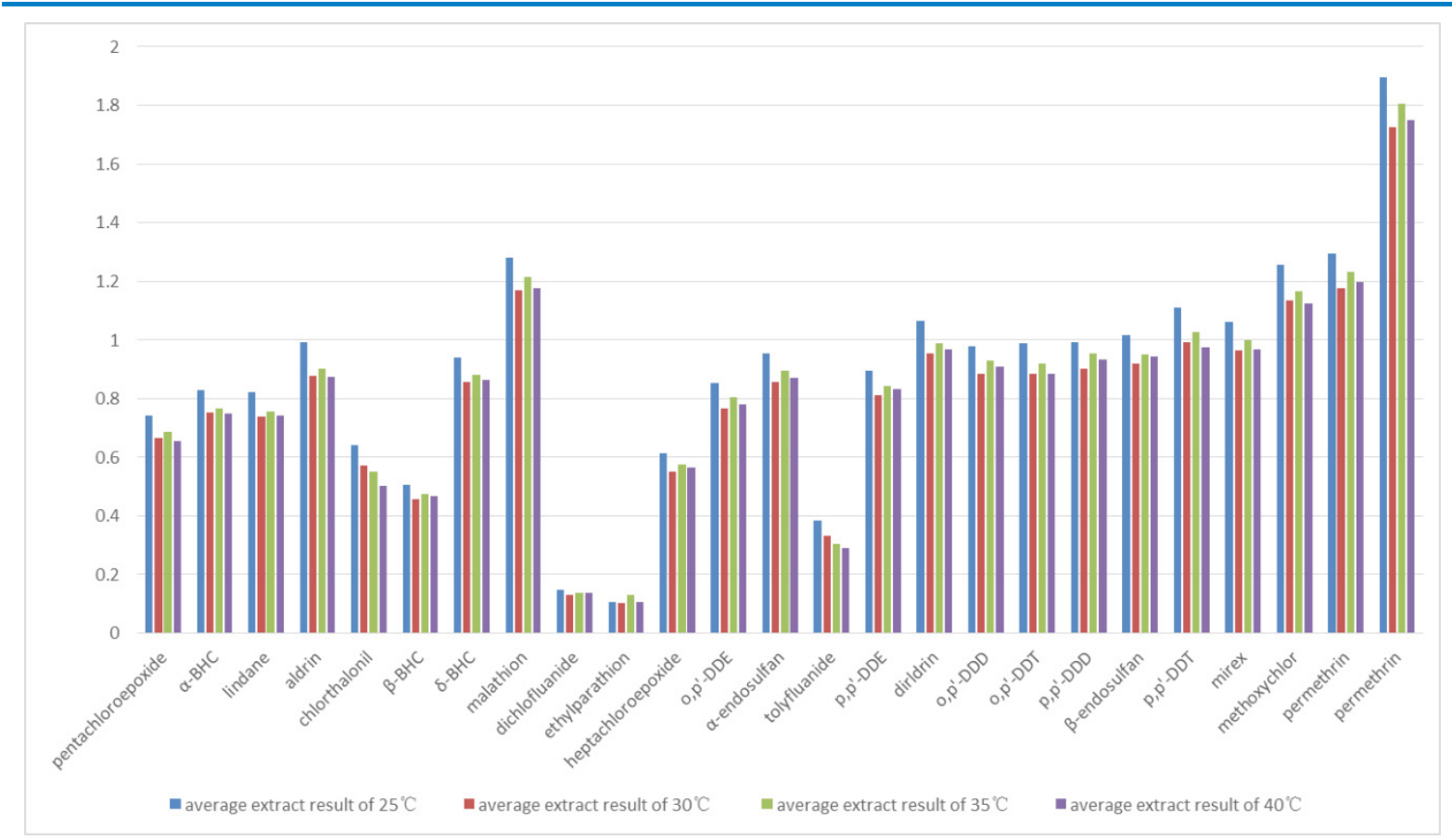

Figure 3. Extraction results at different extraction temperature Linearity and limit of detection (LOD)

A series of mixed standard working solutions $(10,20,50,100,200,500,1000 \mathrm{ng} \mathrm{mL}$ $\left.{ }^{1}\right)$ were prepared and all determined under the optimal pretreatment and instrument conditions. Then the standard curves were plotted between the peak areas and corresponding concentrations and it showed linear regression relationship in the certain range. Limit of detection (LOD) and limit of quantitation (LOQ) were performed using spiked standard samples method and LOQ of the method for the analytes were calculated at signal-to-noise $(\mathrm{S} / \mathrm{N})$ ratio of 3 and 10 . All the above experimental results, retention time, quantitative and qualitative ions and ion abundance ratios of 24 pesticide residues were listed in Table 3.

From the results in Table 3, it was revealed that 24 analytes presented good linearity, with coefficient of determination $\left(R^{2}\right)$ between 0.9983 and 0.9998 . The LODs were in the range of $0.3-15.0 \mathrm{ng} / \mathrm{mL}(0.01-0.15 \mathrm{mg} / \mathrm{kg})$, and the LOQ ranged from 2.0 to $50.0 \mathrm{ng} / \mathrm{mL}$.

\section{Matrix Effects}

For GC-MS analysis, it is necessary to evaluate method matrix effects for each analyte, because they may result from various physical and chemical processes which are difficult to eliminate in analysis. To estimate matrix effects, the slopes of the matrix-matched calibration curves are compared with those obtained in solvent without matrices, and matrix effect (ME\%) is evaluated as the following equation:

$$
\mathrm{ME}(\%)=\mathrm{B} / \mathrm{A} \times 100
$$

where $A$ and $B$ are the slopes of calibration curve without matrix and with different matrix [15-17]. ME\% values suggest that the ionization signals of target compounds were enhanced and suppressed by the matrix, respectively. Generally, ME\% ranging from $85 \%$ to $115 \%$ indicate that the signal enhancement or suppression is acceptable, while ME values $<85 \%$ or $>115 \%$ refer to strong matrix effects.

From Table 3, it was known that ME\% of most of pesticides were between $85 \%$ and $115 \%$ in solvent, blank, cow leather, sheep leather, pig leather, PU and PVC and the matrix effects were very small and could be ignored according to the requirements. The ME\% of malathion, ethylparathion, o, $p^{\prime}-D D D, p, p^{\prime}-D D D$ was more than $115 \%$, and it meant that they had strong matrix enhancement effect. Meanwhile, the ME\% of $\alpha$-endosulfan, $\beta$-endosulfan, o, $p^{\prime}$-DDT, p, $\mathrm{p}^{\prime}-\mathrm{DDT}$, and methoxychlor was less than $85 \%$, and it showed that these analytes had strong signal suppression effect by matrix. To obtain the results more accurately, it was necessary to use the blank negative sample to calibrate the working curve. 
Table 3: Overview of the methodological characteristics including linear range, linear equation, coefficient of determination $\left(R^{2}\right)$, matrix effect $(M E), L O D$, and $L O Q$ for each analyte in different samples

\begin{tabular}{|c|c|c|c|c|c|c|c|c|}
\hline The analyte & $\begin{array}{l}\text { Quantita- } \\
\text { tive ion } \\
\mathrm{m} / \mathrm{z}\end{array}$ & matrix & $\begin{array}{c}\text { Linear } \\
\text { range } \\
(\mathrm{ng} / \mathrm{mL}) \\
\end{array}$ & Linear equation & $\begin{array}{c}\text { Coefficient } \\
\mathrm{R}^{2}\end{array}$ & ME (\%) & $\begin{array}{c}\mathrm{LOD} \\
\mathrm{ng} / \\
\mathrm{mL} \\
\end{array}$ & $\begin{array}{c}\mathrm{LOQ} \\
\mathrm{ng} / \\
\mathrm{mL}\end{array}$ \\
\hline \multirow{8}{*}{$\begin{array}{l}\text { Penta- } \\
\text { chloroanisole } \\
\text { (1) }\end{array}$} & \multirow{8}{*}{280} & solvent & $10^{\sim} 1000$ & $Y=31.58 X+2.004$ & 0.9998 & 100.00 & 1 & 3 \\
\hline & & blank & $10 \sim 1000$ & $Y=30.48 X+229.1$ & 0.9996 & 96.52 & 3 & 10 \\
\hline & & cowhide & 20 1000 & $Y=33.74 X+127.8$ & 0.9978 & 106.84 & 5 & 15 \\
\hline & & $\begin{array}{l}\text { Sheep } \\
\text { leather }\end{array}$ & $20 \sim 1000$ & $Y=27.87 X+345.9$ & 0.9934 & 88.25 & 6 & 20 \\
\hline & & Pig leather & $50 \sim 1000$ & $\mathrm{Y}=27.94 \mathrm{X}+722.1$ & 0.9956 & 88.47 & 10 & 30 \\
\hline & & PU & $20 \sim 1000$ & $Y=29.03 X-738.1$ & 0.9965 & 91.93 & 5 & 15 \\
\hline & & PVC & 20 1000 & $Y=27.98 X+913.3$ & 0.9949 & 88.60 & 5 & 15 \\
\hline & & solvent & $10 \sim 1000$ & $Y=32.17 X-34.99$ & 0.9997 & 100.00 & 2 & 6 \\
\hline \multirow{5}{*}{$\begin{array}{l}\alpha \text {-Benzenehexa- } \\
\text { chloride } \\
(\alpha-B H C, 2)\end{array}$} & \multirow{5}{*}{181} & blank & $20 \sim 1000$ & $\mathrm{Y}=30.68 \mathrm{X}+38.54$ & 0.9974 & 95.37 & 8 & 25 \\
\hline & & cowhide & $50 \sim 1000$ & $Y=35.08 X+39.66$ & 0.9997 & 109.05 & 10 & 30 \\
\hline & & $\begin{array}{l}\text { Sheep } \\
\text { leather }\end{array}$ & $50 \sim 1000$ & $Y=27.85 X+57.12$ & 0.9965 & 86.57 & 15 & 50 \\
\hline & & Pig leather & $50 \sim 1000$ & $Y=27.49 X+331.1$ & 0.9971 & 85.45 & 15 & 50 \\
\hline & & $\mathrm{PU}$ & $50 \sim 1000$ & $Y=35.64 X-1004$ & 0.9971 & 110.79 & 10 & 30 \\
\hline \multirow{9}{*}{$\begin{array}{l}\text { Lindane } \\
\text { (3) }\end{array}$} & \multirow{9}{*}{181} & PVC & $50 \sim 1000$ & $Y=30.52 X+382.7$ & 0.9983 & 94.87 & 10 & 30 \\
\hline & & solvent & $10 \sim 1000$ & $Y=27.36 X-37.28$ & 0.9998 & 100.00 & 2 & 6 \\
\hline & & blank & $20 \sim 1000$ & $Y=25.36 X+163.3$ & 0.9972 & 92.69 & 8 & 25 \\
\hline & & cowhide & $50 \sim 1000$ & $Y=28.67 X-626$ & 0.9989 & 104.79 & 10 & 30 \\
\hline & & $\begin{array}{l}\text { Sheep } \\
\text { leather }\end{array}$ & $50 \sim 1000$ & $Y=27.89 X-424$ & 0.9962 & 101.94 & 15 & 50 \\
\hline & & Pig leather & $50 \sim 1000$ & $Y=26.92 X-81.29$ & 0.9969 & 98.39 & 15 & 50 \\
\hline & & PU & $50 \sim 1000$ & $Y=30.88 X-672$ & 0.9964 & 112.87 & 10 & 30 \\
\hline & & PVC & $50 \sim 1000$ & $Y=24.27 X+570.7$ & 0.9981 & 88.71 & 10 & 30 \\
\hline & & solvent & $10 \sim 1000$ & $Y=18.12 X-23.22$ & 0.9998 & 100.00 & 2 & 6 \\
\hline \multirow{5}{*}{$\begin{array}{l}\text { Aldrin } \\
\text { (4) }\end{array}$} & \multirow{5}{*}{263} & blank & $10 \sim 1000$ & $Y=18.26 X+76.58$ & 0.9981 & 100.77 & 8 & 25 \\
\hline & & cowhide & $50 \sim 1000$ & $Y=20.67 X-60.11$ & 0.9994 & 114.07 & 15 & 50 \\
\hline & & $\begin{array}{l}\text { Sheep } \\
\text { leather }\end{array}$ & $100 \sim 1000$ & $Y=19.87 X+234$ & 0.9924 & 109.66 & 30 & 100 \\
\hline & & Pig leather & $100 \sim 1000$ & $\mathrm{Y}=18.31 \mathrm{X}+73.76$ & 0.9978 & 101.05 & 30 & 100 \\
\hline & & PU & $50 \sim 1000$ & $Y=20.46 X-478.3$ & 0.9972 & 112.91 & 10 & 30 \\
\hline \multirow{9}{*}{$\begin{array}{l}\text { Chlorothalonil } \\
\text { (5) }\end{array}$} & \multirow{9}{*}{266} & PVC & $50 \sim 1000$ & $Y=18.53 X+371$ & 0.9968 & 102.26 & 10 & 30 \\
\hline & & solvent & $50 \sim 1000$ & $Y=23.75 X-1291$ & 0.9978 & 100.00 & 15 & 50 \\
\hline & & blank & $100 \sim 1000$ & $Y=26.09 X-147$ & 0.9989 & 109.85 & 23 & 75 \\
\hline & & cowhide & $100 \sim 1000$ & $Y=27.39 X+5290$ & 0.9903 & 115.33 & 30 & 100 \\
\hline & & $\begin{array}{l}\text { Sheep } \\
\text { leather }\end{array}$ & $100 \sim 1000$ & $Y=19.13 X-1323$ & 0.9931 & 80.55 & 30 & 100 \\
\hline & & Pig leather & $100 \sim 1000$ & $Y=20.38 X+3425$ & 0.9921 & 85.81 & 30 & 100 \\
\hline & & PU & $100 \sim 1000$ & $Y=17.16 X-1467$ & 0.9951 & 72.25 & 23 & 75 \\
\hline & & PVC & $50 \sim 1000$ & $Y=26.38 X-2422$ & 0.9954 & 111.07 & 15 & 50 \\
\hline & & solvent & $10 \sim 1000$ & $Y=25.83 X-61.41$ & 0.9999 & 100.00 & 2 & 6 \\
\hline \multirow{5}{*}{$\begin{array}{l}\beta-B H C \\
\text { (6) }\end{array}$} & \multirow{5}{*}{181} & blank & $50 \sim 1000$ & $\mathrm{Y}=24.88 \mathrm{X}+82.35$ & 0.9981 & 96.32 & 10 & 30 \\
\hline & & cowhide & $50 \sim 1000$ & $Y=28.35 X-11.34$ & 0.9996 & 109.76 & 15 & 50 \\
\hline & & $\begin{array}{l}\text { Sheep } \\
\text { leather }\end{array}$ & $50 \sim 1000$ & $Y=22.64 X-2441$ & 0.9932 & 87.65 & 15 & 50 \\
\hline & & Pig leather & $50 \sim 1000$ & $\mathrm{Y}=23.03 \mathrm{X}+691$ & 0.9954 & 89.16 & 15 & 50 \\
\hline & & $\mathrm{PU}$ & $50 \sim 1000$ & $Y=29.54 X-704.8$ & 0.9967 & 114.36 & 10 & 30 \\
\hline \multirow{8}{*}{$\begin{array}{l}\delta-\mathrm{BHC} \\
(7)\end{array}$} & \multirow{8}{*}{181} & PVC & $50 \sim 1000$ & $Y=22.24 X+684.9$ & 0.9943 & 86.10 & 10 & 30 \\
\hline & & solvent & $10 \sim 1000$ & $Y=23.09 X-106.5$ & 0.9997 & 100.00 & 2 & 6 \\
\hline & & blank & $50 \sim 1000$ & $Y=21.11 X+258.9$ & 0.9951 & 91.42 & 10 & 30 \\
\hline & & cowhide & $50 \sim 1000$ & $Y=25.72 X+443.3$ & 0.9984 & 111.39 & 15 & 50 \\
\hline & & $\begin{array}{l}\text { Sheep } \\
\text { leather }\end{array}$ & $50 \sim 1000$ & $Y=24.71 X+148.4$ & 0.9927 & 107.02 & 15 & 50 \\
\hline & & Pig leather & $50 \sim 1000$ & $Y=21.23 X+868.2$ & 0.9931 & 91.94 & 15 & 50 \\
\hline & & PU & $50 \sim 1000$ & $Y=26.46 X+167.1$ & 0.9964 & 114.60 & 10 & 30 \\
\hline & & PVC & $50 \sim 1000$ & $Y=26.51 X-220$ & 0.9983 & 114.81 & 10 & 30 \\
\hline
\end{tabular}




\begin{tabular}{|c|c|c|c|c|c|c|c|c|}
\hline The analyte & $\begin{array}{l}\text { Quantita- } \\
\text { tive ion } \\
\mathrm{m} / \mathrm{z} \\
\end{array}$ & matrix & $\begin{array}{l}\text { Linear } \\
\text { range } \\
(\mathrm{ng} / \mathrm{mL})\end{array}$ & Linear equation & $\begin{array}{c}\text { Coefficient } \\
\mathrm{R}^{2}\end{array}$ & ME (\%) & $\begin{array}{l}\mathrm{LOD} \\
\mathrm{ng} / \\
\mathrm{mL}\end{array}$ & $\begin{array}{c}\mathrm{LOQ} \\
\mathrm{ng} / \\
\mathrm{mL}\end{array}$ \\
\hline \multirow{7}{*}{$\begin{array}{l}\text { Malathion } \\
\text { (8) }\end{array}$} & \multirow{7}{*}{173} & solvent & $10 \sim 1000$ & $Y=36.24 X-515.1$ & 0.9987 & 100.00 & 2 & 6 \\
\hline & & blank & 20 1000 & $Y=41.95 X+309.1$ & 0.9965 & 115.76 & 6 & 20 \\
\hline & & cowhide & $50 \sim 1000$ & $Y=57.42 X-912.5$ & 0.9987 & 158.44 & 15 & 50 \\
\hline & & $\begin{array}{l}\text { Sheep } \\
\text { leather }\end{array}$ & $100 \sim 1000$ & $Y=45.78 X-893$ & 0.9952 & 126.32 & 20 & 60 \\
\hline & & Pig leather & $100 \sim 1000$ & $Y=46.51 X-1840$ & 0.9957 & 128.34 & 30 & 100 \\
\hline & & PU & $50 \sim 1000$ & $Y=55.75 X-747.1$ & 0.9976 & 153.84 & 10 & 30 \\
\hline & & PVC & 20 1000 & $Y=50.51 X+1106$ & 0.9986 & 139.38 & 6 & 20 \\
\hline \multirow{7}{*}{$\begin{array}{l}\text { Dichlofluanide } \\
\text { (9) }\end{array}$} & \multirow{7}{*}{123} & solvent & $10 \sim 1000$ & $Y=51.09 X-126.4$ & 0.9976 & 100.00 & 3 & 10 \\
\hline & & blank & $50 \sim 1000$ & $Y=43.79 X-1112$ & 0.9961 & 85.71 & 30 & 100 \\
\hline & & cowhide & $100 \sim 1000$ & $Y=60.91 X-1854$ & 0.9988 & 119.22 & 30 & 100 \\
\hline & & $\begin{array}{l}\text { Sheep } \\
\text { leather }\end{array}$ & $100 \sim 1000$ & $Y=45.87 X-1242$ & 0.9967 & 89.78 & 30 & 100 \\
\hline & & Pig leather & $100 \sim 1000$ & $Y=41.87 X+2126$ & 0.9975 & 81.95 & 30 & 100 \\
\hline & & PU & $100 \sim 1000$ & $Y=62.87 X-4773$ & 0.9994 & 123.06 & 30 & 100 \\
\hline & & PVC & $100 \sim 1000$ & $Y=59.14 X-1769$ & 0.9989 & 115.76 & 20 & 60 \\
\hline \multirow{7}{*}{$\begin{array}{l}\text { Ethylparathion } \\
\text { (10) }\end{array}$} & \multirow{7}{*}{275} & solvent & $20 \sim 1000$ & $Y=3.879 X-14.57$ & 0.9976 & 100.00 & 6 & 20 \\
\hline & & blank & 20 1000 & $Y=4.639 X+30.11$ & 0.9943 & 119.59 & 20 & 60 \\
\hline & & cowhide & $50 \sim 1000$ & $Y=8.189 X-42.11$ & 0.9989 & 211.11 & 23 & 75 \\
\hline & & $\begin{array}{l}\text { Sheep } \\
\text { leather }\end{array}$ & $100 \sim 1000$ & $Y=7.231 X-452$ & 0.9936 & 186.41 & 30 & 100 \\
\hline & & Pig leather & $100 \sim 1000$ & $Y=7.041 X+218.6$ & 0.9934 & 181.52 & 30 & 100 \\
\hline & & PU & $100 \sim 1000$ & $Y=7.907 X-267.9$ & 0.9953 & 203.84 & 20 & 60 \\
\hline & & PVC & $50 \sim 1000$ & $Y=7.642 X-33.66$ & 0.9971 & 197.01 & 10 & 30 \\
\hline \multirow{7}{*}{$\begin{array}{l}\text { Hepta- } \\
\text { chloroepoxide } \\
\text { (11) }\end{array}$} & \multirow{7}{*}{353} & solvent & 20 1000 & $Y=3.445 X+1.98$ & 0.9997 & 100.00 & 6 & 20 \\
\hline & & blank & 20 1000 & $Y=3.734 X+3.592$ & 0.9989 & 108.39 & 15 & 50 \\
\hline & & cowhide & $50 \sim 1000$ & $Y=3.926 X-4.895$ & 0.9992 & 113.96 & 15 & 50 \\
\hline & & $\begin{array}{l}\text { Sheep } \\
\text { leather }\end{array}$ & $50 \sim 1000$ & $Y=3.532 X+32.56$ & 0.9974 & 102.53 & 15 & 50 \\
\hline & & Pig leather & $50 \sim 1000$ & $Y=3.405 X+42.79$ & 0.9962 & 98.84 & 15 & 50 \\
\hline & & PU & $50 \sim 1000$ & $Y=3.489 X-90.61$ & 0.9959 & 101.28 & 10 & 30 \\
\hline & & PVC & $50 \sim 1000$ & $Y=3.567 X+77.34$ & 0.9981 & 103.54 & 10 & 30 \\
\hline \multirow{7}{*}{$\begin{array}{l}\text { o,p'dichloro } \\
\text { (diphenyl) } \\
\text { ethylene } \\
\text { (o,p'-DDE, 12) }\end{array}$} & \multirow{7}{*}{246} & solvent & $10 \sim 1000$ & $Y=84.93 X-81.78$ & 0.9998 & 100.00 & 0.3 & 1 \\
\hline & & blank & $10 \sim 1000$ & $Y=86.34 X+304.3$ & 0.9981 & 101.66 & 3 & 10 \\
\hline & & cowhide & $10 \sim 1000$ & $Y=95.54 X-156.8$ & 0.9996 & 112.49 & 3 & 10 \\
\hline & & $\begin{array}{l}\text { Sheep } \\
\text { leather }\end{array}$ & $20 \sim 1000$ & $Y=84.67 X-245$ & 0.9972 & 99.69 & 3 & 10 \\
\hline & & Pig leather & 20 1000 & $Y=88.01 X+789.3$ & 0.9966 & 103.63 & 3 & 10 \\
\hline & & PU & $10 \sim 1000$ & $Y=79.06 X+570.1$ & 0.9923 & 93.09 & 3 & 10 \\
\hline & & PVC & $10 \sim 1000$ & $Y=87.77 X+3034$ & 0.9985 & 103.34 & 3 & 10 \\
\hline \multirow{7}{*}{$\begin{array}{l}\alpha \text {-Endosulfan } \\
\text { (13) }\end{array}$} & \multirow{7}{*}{241} & solvent & $50 \sim 1000$ & $Y=3.992 X+17.7$ & 0.9998 & 100.00 & 8 & 25 \\
\hline & & blank & $50 \sim 1000$ & $Y=4.263 X+182.2$ & 0.9986 & 106.79 & 30 & 100 \\
\hline & & cowhide & $100 \sim 1000$ & $Y=3.156 X-243.9$ & 0.9966 & 79.06 & 30 & 100 \\
\hline & & $\begin{array}{l}\text { Sheep } \\
\text { leather }\end{array}$ & $100 \sim 1000$ & $Y=3.032 X+563$ & 0.9951 & 75.95 & 30 & 100 \\
\hline & & Pig leather & $100 \sim 1000$ & $Y=2.681 X+244.7$ & 0.9943 & 67.16 & 30 & 100 \\
\hline & & $\mathrm{PU}$ & $100 \sim 1000$ & $Y=3.606 X+207$ & 0.9944 & 90.33 & 30 & 100 \\
\hline & & PVC & $100 \sim 1000$ & $Y=4.235 X+152.1$ & 0.9962 & 106.09 & 30 & 100 \\
\hline \multirow{7}{*}{$\begin{array}{l}\text { Tolyfluanide } \\
\text { (14) }\end{array}$} & \multirow{7}{*}{238} & solvent & $10 \sim 1000$ & $Y=21.51 X-465.9$ & 0.9978 & 100.00 & 3 & 10 \\
\hline & & blank & $50 \sim 1000$ & $Y=20.53 X-445$ & 0.9988 & 95.44 & 15 & 50 \\
\hline & & cowhide & $100 \sim 1000$ & $Y=22.39 X-181.1$ & 0.9987 & 104.09 & 30 & 100 \\
\hline & & $\begin{array}{l}\text { Sheep } \\
\text { leather }\end{array}$ & $100 \sim 1000$ & $Y=21.63 X-242.1$ & 0.9963 & 100.56 & 30 & 100 \\
\hline & & Pig leather & $100 \sim 1000$ & $Y=20.89 X+170.8$ & 0.9939 & 97.12 & 30 & 100 \\
\hline & & $\mathrm{PU}$ & $50 \sim 1000$ & $Y=21.89 X-6996$ & 0.9972 & 101.77 & 15 & 50 \\
\hline & & PVC & $50 \sim 1000$ & $Y=24.55 X-159.4$ & 0.9973 & 114.13 & 10 & 30 \\
\hline
\end{tabular}




\begin{tabular}{|c|c|c|c|c|c|c|c|c|}
\hline The analyte & $\begin{array}{l}\text { Quantita- } \\
\text { tive ion } \\
\mathrm{m} / \mathrm{z} \\
\end{array}$ & matrix & $\begin{array}{l}\text { Linear } \\
\text { range } \\
\text { (ng/mL) }\end{array}$ & Linear equation & $\begin{array}{c}\text { Coefficient } \\
\mathrm{R}^{2}\end{array}$ & ME (\%) & $\begin{array}{c}\mathrm{LOD} \\
\mathrm{ng} / \\
\mathrm{mL} \\
\end{array}$ & $\begin{array}{c}\mathrm{LOQ} \\
\mathrm{ng} / \\
\mathrm{mL} \\
\end{array}$ \\
\hline \multirow{7}{*}{$\begin{array}{l}p, p^{\prime}-D D E \\
(15)\end{array}$} & \multirow{6}{*}{318} & solvent & $10^{\sim} 1000$ & $Y=51.26 X-53.69$ & 0.9997 & 100.00 & 0.6 & 2 \\
\hline & & blank & $10 \sim 1000$ & $Y=54.29 X+105.1$ & 0.9991 & 105.91 & 1 & 3 \\
\hline & & cowhide & $10 \sim 1000$ & $Y=56.69 X+2.21$ & 0.9996 & 110.59 & 3 & 10 \\
\hline & & $\begin{array}{l}\text { Sheep } \\
\text { leather }\end{array}$ & $10 \sim 1000$ & $Y=42.89 X+345$ & 0.9962 & 83.67 & 3 & 10 \\
\hline & & Pig leather & $10 \sim 1000$ & $Y=43.47 X+1612$ & 0.9938 & 84.80 & 3 & 10 \\
\hline & & PU & $10 \sim 1000$ & $Y=48.58 X+272.8$ & 0.9932 & 94.77 & 2 & 6 \\
\hline & & PVC & $10 \sim 1000$ & $Y=45.47 X+1791$ & 0.9934 & 88.70 & 2 & 6 \\
\hline \multirow{7}{*}{$\begin{array}{l}\text { Dieldrin } \\
\text { (16) }\end{array}$} & \multirow{7}{*}{263} & solvent & $10 \sim 1000$ & $Y=8.776 X+51.73$ & 0.9995 & 100.00 & 4 & 10 \\
\hline & & blank & $10 \sim 1000$ & $Y=9.157 X+50.29$ & 0.9953 & 104.34 & 15 & 50 \\
\hline & & cowhide & $50 \sim 1000$ & $Y=7.971 X-495.6$ & 0.9924 & 90.83 & 15 & 50 \\
\hline & & $\begin{array}{l}\text { Sheep } \\
\text { leather }\end{array}$ & $50 \sim 1000$ & $Y=7.672 X+241$ & 0.9935 & 87.42 & 15 & 50 \\
\hline & & Pig leather & $50 \sim 1000$ & $Y=8.471 X+1.368$ & 0.9976 & 96.52 & 15 & 50 \\
\hline & & PU & $50 \sim 1000$ & $Y=8.522 X-32.01$ & 0.9931 & 97.11 & 15 & 50 \\
\hline & & PVC & $50 \sim 1000$ & $Y=10.11 X+1.474$ & 0.9981 & 115.20 & 15 & 50 \\
\hline \multirow{7}{*}{$\begin{array}{l}\text { o,p'-dichloro } \\
\text { bis(4- } \\
\text { chlorophenyl) } \\
\text { ethane } \\
\left(0, p^{\prime}-D D D, 17\right)\end{array}$} & \multirow{6}{*}{235} & solvent & $10 \sim 1000$ & $Y=97.91 X-150$ & 0.9997 & 100.00 & 0.6 & 2 \\
\hline & & blank & $10 \sim 1000$ & $Y=113.4 X+243.5$ & 0.9989 & 115.82 & 2 & 6 \\
\hline & & cowhide & $20 \sim 1000$ & $Y=122.5 X-410.7$ & 0.9997 & 125.11 & 6 & 20 \\
\hline & & $\begin{array}{l}\text { Sheep } \\
\text { leather }\end{array}$ & $50 \sim 1000$ & $Y=117.3 X-652$ & 0.9954 & 119.80 & 10 & 30 \\
\hline & & Pig leather & $50 \sim 1000$ & $Y=121.8 X+513.2$ & 0.9964 & 124.40 & 10 & 30 \\
\hline & & PU & $10 \sim 1000$ & $Y=121.3 X+496.6$ & 0.9933 & 123.89 & 2 & 6 \\
\hline & \multirow{8}{*}{235} & PVC & $10 \sim 1000$ & $Y=120.7 X+3018$ & 0.9974 & 123.28 & 2 & 6 \\
\hline \multirow{7}{*}{$\begin{array}{l}o, p^{\prime} \text {-dichloro } \\
\text { (diphenyl) } \\
\text { trichloroethane } \\
\text { (o,p'-DDT, 18) }\end{array}$} & & solvent & $10 \sim 1000$ & $Y=76.68 X-1140$ & 0.9989 & 100.00 & 1 & 3 \\
\hline & & blank & $10 \sim 1000$ & $Y=71.71 X+306.4$ & 0.9981 & 93.52 & 3 & 10 \\
\hline & & cowhide & $20 \sim 1000$ & $Y=77.69 X+138.3$ & 0.9998 & 101.32 & 5 & 15 \\
\hline & & $\begin{array}{l}\text { Sheep } \\
\text { leather }\end{array}$ & $50 \sim 1000$ & $Y=54.82 X+2834$ & 0.9931 & 71.49 & 10 & 30 \\
\hline & & Pig leather & $50 \sim 1000$ & $Y=51.02 X+2938$ & 0.9929 & 66.54 & 10 & 30 \\
\hline & & PU & $20 \sim 1000$ & $Y=68.87 X+606.4$ & 0.9934 & 89.81 & 5 & 15 \\
\hline & & PVC & $10 \sim 1000$ & $Y=54.46 X+1291$ & 0.9926 & 71.02 & 3 & 10 \\
\hline \multirow{7}{*}{$\begin{array}{l}p, p^{\prime}-D D D \\
(19)\end{array}$} & \multirow{6}{*}{235} & solvent & $10 \sim 1000$ & $Y=106.1 X-335$ & 0.9997 & 100.00 & 0.6 & 2 \\
\hline & & blank & $10 \sim 1000$ & $Y=127.3 X+225.9$ & 0.9984 & 119.98 & 2 & 6 \\
\hline & & cowhide & $10 \sim 1000$ & $Y=133.5 X-87.84$ & 0.9998 & 125.82 & 3 & 10 \\
\hline & & $\begin{array}{l}\text { Sheep } \\
\text { leather }\end{array}$ & $50 \sim 1000$ & $Y=132.4 X+4522$ & 0.9935 & 124.79 & 10 & 30 \\
\hline & & Pig leather & $50 \sim 1000$ & $Y=126.1 X+2374$ & 0.9925 & 118.85 & 10 & 30 \\
\hline & & PU & $10 \sim 1000$ & $Y=116.7 X+334$ & 0.9937 & 109.99 & 2 & 6 \\
\hline & \multirow{8}{*}{241} & PVC & $10 \sim 1000$ & $Y=120.3 X+5296$ & 0.9944 & 113.38 & 2 & 6 \\
\hline \multirow{7}{*}{$\begin{array}{l}\beta \text {-Endosulfan } \\
\text { (20) }\end{array}$} & & solvent & $50 \sim 1000$ & $\mathrm{Y}=2.642 \mathrm{X}+120.1$ & 0.9991 & 100.00 & 15 & 50 \\
\hline & & blank & 50 1000 & $Y=2.151 X+616.6$ & 0.9955 & 81.42 & 15 & 50 \\
\hline & & cowhide & $100 \sim 1000$ & $Y=2.127 X-73.08$ & 0.9905 & 80.51 & 30 & 100 \\
\hline & & $\begin{array}{l}\text { Sheep } \\
\text { leather }\end{array}$ & $100 \sim 1000$ & $Y=2.034 X+1312$ & 0.9902 & 76.99 & 30 & 100 \\
\hline & & Pig leather & $100 \sim 1000$ & $Y=1.903 X+1049$ & 0.9918 & 72.03 & 30 & 100 \\
\hline & & PU & $100 \sim 1000$ & $Y=1.797 X+1765$ & 0.9913 & 68.02 & 20 & 60 \\
\hline & & PVC & $100 \sim 1000$ & $Y=2.253 X+390$ & 0.9979 & 85.28 & 20 & 60 \\
\hline \multirow{7}{*}{$\begin{array}{l}p, p^{\prime}-D D T \\
(21)\end{array}$} & \multirow{7}{*}{235} & solvent & $10 \sim 1000$ & $Y=77.75 X-1764$ & 0.9976 & 100.00 & 1 & 3 \\
\hline & & blank & $10 \sim 1000$ & $Y=67.17 X+223.9$ & 0.9964 & 86.39 & 3 & 10 \\
\hline & & cowhide & $10 \sim 1000$ & $Y=67.99 X+1329$ & 0.9997 & 87.45 & 3 & 10 \\
\hline & & $\begin{array}{l}\text { Sheep } \\
\text { leather }\end{array}$ & $50 \sim 1000$ & $Y=53.45 X+5631$ & 0.9937 & 68.75 & 10 & 30 \\
\hline & & Pig leather & $50 \sim 1000$ & $Y=44.01 X+6144$ & 0.9957 & 56.60 & 10 & 30 \\
\hline & & PU & $10 \sim 1000$ & $Y=66.03 X+289.5$ & 0.9925 & 84.93 & 3 & 10 \\
\hline & & PVC & $10 \sim 1000$ & $Y=47.62 X+1058$ & 0.9929 & 61.25 & 3 & 10 \\
\hline
\end{tabular}




\begin{tabular}{|c|c|c|c|c|c|c|c|c|}
\hline The analyte & $\begin{array}{c}\text { Quantita- } \\
\text { tive ion } \\
\mathrm{m} / \mathrm{z} \\
\end{array}$ & matrix & $\begin{array}{c}\text { Linear } \\
\text { range } \\
\text { (ng/mL) }\end{array}$ & Linear equation & $\begin{array}{c}\text { Coefficient } \\
\mathrm{R}^{2}\end{array}$ & ME (\%) & $\begin{array}{c}\text { LOD } \\
\mathrm{ng} / \\
\mathrm{mL}\end{array}$ & $\begin{array}{c}\mathrm{LOQ} \\
\mathrm{ng} / \\
\mathrm{mL}\end{array}$ \\
\hline \multirow{7}{*}{$\begin{array}{l}\text { Mirex } \\
(22)\end{array}$} & \multirow{7}{*}{272} & solvent & $10 \sim 1000$ & $Y=48.08 X+22.72$ & 0.9996 & 100.00 & 3 & 10 \\
\hline & & blank & $10 \sim 1000$ & $Y=47.28 X+62.23$ & 0.9985 & 98.34 & 3 & 10 \\
\hline & & cowhide & $10 \sim 1000$ & $Y=41.22 X-164.1$ & 0.9998 & 85.73 & 3 & 10 \\
\hline & & $\begin{array}{l}\text { Sheep } \\
\text { leather }\end{array}$ & $20 \sim 1000$ & $Y=43.67 X+452$ & 0.9978 & 90.83 & 6 & 20 \\
\hline & & Pig leather & $20 \sim 1000$ & $Y=42.76 X+649.2$ & 0.9961 & 88.94 & 6 & 20 \\
\hline & & PU & $10 \sim 1000$ & $Y=41.98 X+268.6$ & 0.9934 & 87.31 & 3 & 10 \\
\hline & & PVC & $10 \sim 1000$ & $Y=44.73 X+961.3$ & 0.9972 & 93.03 & 3 & 10 \\
\hline \multirow{7}{*}{$\begin{array}{l}\text { Methoxychlor } \\
\text { (23) }\end{array}$} & \multirow{7}{*}{227} & solvent & $10 \sim 1000$ & $Y=140.4 X-2725$ & 0.9983 & 100.00 & 0.6 & 2 \\
\hline & & blank & $10 \sim 1000$ & $Y=126.4 X+98.56$ & 0.9985 & 90.03 & 2 & 6 \\
\hline & & cowhide & $10 \sim 1000$ & $Y=129.1 X+1090$ & 0.9998 & 91.95 & 3 & 10 \\
\hline & & $\begin{array}{l}\text { Sheep } \\
\text { leather }\end{array}$ & 20 1000 & $Y=132.4 X+1343$ & 0.9976 & 94.30 & 6 & 20 \\
\hline & & Pig leather & 20 1000 & $Y=102.8 X+6422$ & 0.9935 & 73.22 & 6 & 20 \\
\hline & & $\mathrm{PU}$ & $10 \sim 1000$ & $Y=117.1 X+1178$ & 0.9942 & 83.40 & 3 & 10 \\
\hline & & PVC & $10 \sim 1000$ & $Y=110.4 X+265.1$ & 0.9983 & 78.63 & 2 & 6 \\
\hline \multirow{7}{*}{$\begin{array}{l}\text { Permethrin } \\
\text { (24) }\end{array}$} & \multirow{7}{*}{183} & solvent & $10 \sim 1000$ & $Y=116.6 X+26.87$ & 0.9998 & 100.00 & 3 & 10 \\
\hline & & blank & $10 \sim 1000$ & $Y=128.1 X+622.8$ & 0.9952 & 109.86 & 3 & 10 \\
\hline & & cowhide & 20 1000 & $Y=125.2 X+1069$ & 0.9999 & 107.38 & 5 & 15 \\
\hline & & $\begin{array}{l}\text { Sheep } \\
\text { leather }\end{array}$ & 20 1000 & $Y=127.7 X+2234$ & 0.9987 & 109.52 & 6 & 20 \\
\hline & & Pig leather & 20 1000 & $Y=119.5 X+7337$ & 0.9977 & 102.49 & 6 & 20 \\
\hline & & PU & $20 \sim 1000$ & $Y=111.9 X+1962$ & 0.9937 & 95.97 & 6 & 20 \\
\hline & & PVC & $10 \sim 1000$ & $Y=124.4 X+3987$ & 0.9992 & 106.69 & 3 & 10 \\
\hline
\end{tabular}

\section{Recovery and Precision Test}

Blank negative leather samples (including cow leather, sheep leather, and pig leather) at $100,300,600 \mu \mathrm{g} / \mathrm{kg}$ levels of mixed standard solutions were selected to perform the recovery experiment. Precisions were evaluated by using the relative standard deviation (RSD) of six measurements. The average recoveries for 24 analytes were in the range of $75.49 \%-131 \%$. The precision was $1.14 \%-14.37 \%$. The experimental results were shown in supplemental materials (Tables S2A, S2B and S2C). These results can satisfy the requirement of testing the pesticide residues in leather product.

\section{Determination of 24 Pesticide Residues in Leather Samples}

The developed and validated method was applied to detect the presence and quantify 24 pesticide residues in three positive samples. Results were shown in Table 4. The experimental results showed that the concentrations of most pesticide residues in these samples were about 200,500,1000 $\mathrm{gg} / \mathrm{kg}$. And the RSD was the range of $2 \% \sim 13 \%$. However, chlorothalonil, dichlofluanide, and tolyfluanide were not detected because of degradation.

Table 4: The average content of 24 pesticide residues in three positive leather samples

\begin{tabular}{|c|c|c|c|c|c|c|c|c|c|}
\hline \multirow{2}{*}{ Analyte } & \multirow{2}{*}{$\begin{array}{l}\text { Sample } \\
\text { number }\end{array}$} & \multicolumn{6}{|c|}{ Content $(\mu \mathrm{g} / \mathrm{kg})$} & \multirow{2}{*}{ Average $(\mu \mathrm{g} / \mathrm{kg})$} & \multirow{2}{*}{ RSD (\%) } \\
\hline & & 1 & 2 & 3 & 4 & 5 & 6 & & \\
\hline \multirow{3}{*}{1} & $4 \#$ & 205.7 & 216.7 & 194.2 & 198.7 & 204.3 & 197.3 & 202.8 & 3.97 \\
\hline & $5 \#$ & 508.6 & 482.6 & 486.4 & 493.1 & 477.6 & 493.1 & 490.2 & 2.21 \\
\hline & $6 \#$ & 949.3 & 953.7 & 1015.3 & 986.1 & 948.7 & 986.1 & 973.2 & 2.78 \\
\hline \multirow{3}{*}{2} & $4 \#$ & 186.1 & 176 & 176.1 & 182.7 & 177.4 & 194.2 & 182.1 & 3.95 \\
\hline & $5 \#$ & 417.3 & 443.9 & 417.7 & 487.5 & 480.7 & 404.8 & 442.0 & 7.94 \\
\hline & $6 \#$ & 932.2 & 932.9 & 1079 & 986.5 & 938 & 977.2 & 974.3 & 5.79 \\
\hline \multirow{3}{*}{3} & $4 \#$ & 197.4 & 173.7 & 166.4 & 172.5 & 209.3 & 163.4 & 180.5 & 10.27 \\
\hline & $5 \#$ & 463.2 & 438 & 450.6 & 416.6 & 450.8 & 418.6 & 439.6 & 4.29 \\
\hline & $6 \#$ & 818.5 & 1006.9 & 1044 & 1025.4 & 1001.9 & 1025.4 & 987.0 & 8.50 \\
\hline
\end{tabular}




\begin{tabular}{|c|c|c|c|c|c|c|c|c|c|}
\hline \multirow{2}{*}{ Analyte } & \multirow{2}{*}{$\begin{array}{l}\text { Sample } \\
\text { number }\end{array}$} & \multicolumn{6}{|c|}{ Content $(\mu \mathrm{g} / \mathrm{kg})$} & \multirow{2}{*}{ Average $(\mu \mathrm{g} / \mathrm{kg})$} & \multirow{2}{*}{ RSD (\%) } \\
\hline & & 1 & 2 & 3 & 4 & 5 & 6 & & \\
\hline \multirow{3}{*}{4} & $4 \#$ & 219.4 & 213.2 & 225.2 & 213 & 211.7 & 213.4 & 216.0 & 2.43 \\
\hline & $5 \#$ & 557.8 & 543.4 & 542 & 542.8 & 538.4 & 542.8 & 544.5 & 1.24 \\
\hline & $6 \#$ & 1072.3 & 1069.3 & 1167.9 & 1142.2 & 1064.3 & 1142.2 & 1109.7 & 4.15 \\
\hline \multirow{3}{*}{5} & $4 \#$ & -- & -- & -- & -- & -- & -- & -- & -- \\
\hline & $5 \#$ & -- & -- & -- & -- & -- & -- & -- & -- \\
\hline & $6 \#$ & -- & -- & -- & -- & -- & -- & -- & -- \\
\hline \multirow{3}{*}{6} & $4 \#$ & 213.7 & 191.3 & 201.2 & 188.2 & 211.6 & 162.4 & 194.7 & 9.71 \\
\hline & $5 \#$ & 451.1 & 424.8 & 408.8 & 445.4 & 367 & 445.4 & 423.8 & 7.56 \\
\hline & $6 \#$ & 1142.5 & 925.2 & 903 & 1151.5 & 892.6 & 863.1 & 979.7 & 13.39 \\
\hline \multirow{3}{*}{7} & $4 \#$ & 145.8 & 165.4 & 143.8 & 140.2 & 120.6 & 136.2 & 142.0 & 10.25 \\
\hline & $5 \#$ & 298.6 & 229.8 & 220.3 & 254.1 & 224.8 & 254.1 & 247.0 & 11.83 \\
\hline & $6 \#$ & 563.6 & 606.7 & 665.2 & 683.7 & 601.7 & 683.7 & 634.1 & 7.93 \\
\hline \multirow{3}{*}{8} & $4 \#$ & 82 & 86.3 & 72.8 & 75.5 & 58.7 & 81.4 & 76.1 & 12.89 \\
\hline & $5 \#$ & 171.4 & 192.7 & 194 & 191.6 & 187.7 & 191.6 & 188.2 & 4.51 \\
\hline & $6 \#$ & 540.5 & 537.6 & 521.6 & 569.9 & 532.6 & 569.9 & 545.4 & 3.68 \\
\hline \multirow{3}{*}{9} & $4 \#$ & -- & -- & -- & -- & -- & -- & -- & -- \\
\hline & $5 \#$ & -- & -- & -- & -- & -- & -- & -- & -- \\
\hline & $6 \#$ & -- & -- & -- & -- & -- & -- & -- & -- \\
\hline \multirow{3}{*}{10} & $4 \#$ & 54.65 & 56.46 & 50.5 & 44.85 & 58.61 & 65.6 & 55.1 & 12.85 \\
\hline & $5 \#$ & 90.2 & 79.5 & 84.4 & 78.9 & 74.5 & 78.9 & 81.1 & 6.75 \\
\hline & $6 \#$ & 229.3 & 230.4 & 248.4 & 242.4 & 225.4 & 242.4 & 236.4 & 3.89 \\
\hline \multirow{3}{*}{11} & $4 \#$ & 178 & 170.6 & 143.2 & 150.7 & 155.7 & 150.9 & 158.2 & 8.42 \\
\hline & $5 \#$ & 390.5 & 402.2 & 437.4 & 407.9 & 397.2 & 407.9 & 407.2 & 3.99 \\
\hline & $6 \#$ & 872.3 & 854 & 928.5 & 930.7 & 849 & 930.7 & 894.2 & 4.47 \\
\hline \multirow{3}{*}{12} & $4 \#$ & 178.6 & 174.9 & 175.5 & 166.2 & 169.5 & 168.2 & 172.2 & 2.83 \\
\hline & $5 \#$ & 447.3 & 441.7 & 480.6 & 464.3 & 436.7 & 464.3 & 455.8 & 3.67 \\
\hline & $6 \#$ & 945.7 & 947 & 1025.3 & 997.1 & 942 & 997.1 & 975.7 & 3.62 \\
\hline \multirow{3}{*}{13} & $4 \#$ & 336.2 & 271.2 & 351.8 & 307 & 325 & 291.1 & 313.7 & 9.51 \\
\hline & $5 \#$ & 582.5 & 659 & 715.2 & 587.7 & 654 & 587.7 & 631.0 & 8.54 \\
\hline & $6 \#$ & 1211.2 & 1222.2 & 1347.2 & 1249.4 & 1217.2 & 1249.4 & 1249.4 & 4.05 \\
\hline \multirow{3}{*}{14} & $4 \#$ & -- & -- & -- & -- & -- & -- & -- & -- \\
\hline & $5 \#$ & -- & -- & -- & -- & -- & -- & -- & -- \\
\hline & $6 \#$ & -- & -- & -- & -- & -- & -- & -- & -- \\
\hline \multirow{3}{*}{15} & $4 \#$ & 151.1 & 145.2 & 141 & 126.4 & 134.3 & 126.6 & 137.4 & 7.34 \\
\hline & $5 \#$ & 364.5 & 359.4 & 398.2 & 389 & 354.4 & 389 & 375.8 & 4.91 \\
\hline & $6 \#$ & 943.4 & 945.1 & 1006.5 & 972 & 940.1 & 972 & 963.2 & 2.66 \\
\hline \multirow{3}{*}{16} & $4 \#$ & 234.4 & 227.9 & 170.7 & 184.6 & 221.7 & 218.3 & 209.6 & 12.28 \\
\hline & $5 \#$ & 524 & 526.2 & 566.4 & 574.5 & 521.2 & 570.7 & 547.2 & 4.71 \\
\hline & $6 \#$ & 1054.2 & 1036.3 & 1098.5 & 1095 & 1047.7 & 1103.5 & 1072.5 & 2.77 \\
\hline \multirow{3}{*}{17} & $4 \#$ & 169.6 & 168.1 & 162.4 & 155.8 & 159.4 & 157.9 & 162.2 & 3.45 \\
\hline & $5 \#$ & 443.1 & 436.7 & 463.4 & 443.7 & 423 & 454.3 & 444.0 & 3.15 \\
\hline & $6 \#$ & 864.5 & 863.4 & 909.1 & 881.2 & 858.4 & 881.2 & 876.3 & 2.13 \\
\hline
\end{tabular}




\begin{tabular}{|c|c|c|c|c|c|c|c|c|c|}
\hline \multirow{2}{*}{ Analyte } & \multirow{2}{*}{$\begin{array}{l}\text { Sample } \\
\text { number }\end{array}$} & \multicolumn{6}{|c|}{ Content $(\mu \mathrm{g} / \mathrm{kg})$} & \multirow{2}{*}{ Average $(\mu \mathrm{g} / \mathrm{kg})$} & \multirow{2}{*}{ RSD (\%) } \\
\hline & & 1 & 2 & 3 & 4 & 5 & 6 & & \\
\hline \multirow{3}{*}{18} & $4 \#$ & 178.3 & 184.3 & 180.2 & 179 & 179.9 & 179.7 & 180.2 & 1.17 \\
\hline & $5 \#$ & 453.4 & 444 & 504.3 & 500.3 & 439 & 500.3 & 473.6 & 6.58 \\
\hline & $6 \#$ & 1126.5 & 1117.1 & 1196.3 & 1155.5 & 1112.1 & 1155.5 & 1143.8 & 2.78 \\
\hline \multirow{3}{*}{19} & $4 \#$ & 177.6 & 171.3 & 160 & 157.8 & 167.4 & 157.1 & 165.2 & 5.02 \\
\hline & $5 \#$ & 439.8 & 427.9 & 455 & 443.6 & 422.9 & 443.6 & 438.8 & 2.66 \\
\hline & $6 \#$ & 708.1 & 690.8 & 768.6 & 687.1 & 685.8 & 687.1 & 704.6 & 4.61 \\
\hline \multirow{3}{*}{20} & $4 \#$ & 548 & 440.2 & 399.1 & 519.8 & 497.4 & 491 & 482.6 & 11.24 \\
\hline & $5 \#$ & 1213.1 & 1135 & 1085.6 & 1206.9 & 1091.4 & 1162.8 & 1149.1 & 4.80 \\
\hline & $6 \#$ & 2080.3 & 2022.6 & 2200.3 & 1979.2 & 2064.1 & 2025.3 & 2062.0 & 3.71 \\
\hline \multirow{3}{*}{21} & $4 \#$ & 215.4 & 226.9 & 181.5 & 209.6 & 202.4 & 210 & 207.6 & 7.31 \\
\hline & $5 \#$ & 571.2 & 580.9 & 649.8 & 638.6 & 575.9 & 638.6 & 609.2 & 6.02 \\
\hline & $6 \#$ & 1537 & 1511.5 & 1582.1 & 1515.5 & 1506.5 & 1515.5 & 1528.0 & 1.86 \\
\hline \multirow{3}{*}{22} & 4\# & 186.5 & 181 & 174.4 & 163.9 & 177.6 & 164 & 174.6 & 5.24 \\
\hline & $5 \#$ & 431.1 & 425.1 & 481.9 & 475.5 & 420.1 & 475.5 & 451.5 & 6.40 \\
\hline & $6 \#$ & 1010.9 & 1005.5 & 1059.4 & 1019.8 & 1000.5 & 1019.8 & 1019.3 & 2.07 \\
\hline \multirow{3}{*}{23} & $4 \#$ & 180.3 & 188.4 & 181 & 171 & 181.4 & 176 & 179.7 & 3.25 \\
\hline & $5 \#$ & 449.1 & 446.4 & 481.8 & 478.3 & 441.4 & 478.3 & 462.6 & 4.05 \\
\hline & $6 \#$ & 979 & 963.7 & 1009.6 & 983.4 & 958.7 & 983.4 & 979.6 & 1.84 \\
\hline \multirow{3}{*}{24} & $4 \#$ & 223.97 & 213.12 & 204.5 & 208.5 & 220.44 & 205.91 & 212.7 & 3.75 \\
\hline & $5 \#$ & 514.36 & 502.69 & 568.55 & 562.16 & 497.69 & 562.16 & 534.6 & 6.18 \\
\hline & $6 \#$ & 1175 & 1178.6 & 1198.2 & 1153.3 & 1173.6 & 1153.3 & 1172.0 & 1.45 \\
\hline
\end{tabular}

\section{CONCLUSIONS}

This study contributed to a validated method detection standard for quantitative detecting common pesticide residues in leather. Small sample volumes, solid phase extraction and GC-MS was used to identify these 24 pesticide residues at trace concentrations. The method established has been successfully applied in detecting the pesticide residues in cowhide, sheep leather and pig leather. In the future, the supervisor can use this method to monitor the content of the harmful chemicals. It will gain more practical applications in shoe materials and provide the reference and advises for the detection of these pesticides residues in other industry products.

\section{Author Contributions}

Method development, Jinlan Dai and Honglei Yin; Experiment, Jinlan Dai and Hang Wei; Application, Jinlan Dai, and Lei Zhou, Director, Minghua Liu.

\section{Acknowledgement}

This project was financially supported by the key project of Fuzhou Customs District of P.R. China (FK2013-42) and the project of General Administration of Customs of P.R. China (2016IK038), and Natural Science Foundation of Tianjin, China (General Program, No. 14JCYBJC16800).

\section{REFERENCES}

1. Kim, W., Lee, Y., Lee, J.H., Shin, G.W., Yun, M.H., A comparative study on designer and customer preference models of leather for vehicle, Int J Ind Ergonom, 2018, 65, 110-121, https://doi. org/10.1016/j.ergon.2017.07.009.

2. Adigüzel Zengin, A.C., Örk, N., Yiğit, Ü., Bitlisli, B.O., Leather as a potential liner for the prosthetic leg users, Leather and Footwear Journal, 2018, 18, 4, 321-326, https://doi. org/10.24264/lfj.18.4.7. 
3. Indian Leather Group, Furniture and Automotive Leathers, 2015, 49, 5-8.

4. Kvitko, K., Bandinelli, E., Henriques, J.A.P., Heuser, V.D., Rohr, P., da Silva, F.R., Schneider, N.B., Fernandes, S., Ancines, C., da Silva, J., Susceptibility to DNA damage in workers occupationally exposed to pesticides, to tannery chemicals and to coal dust during mining, Genet Mol Biol, 2012, 35, 4 Suppl, 1060-1068, https://doi.org/10.1590\%2 Fs1415-47572012000600022.

5. Lin, F., Chen, X.C., Li, X.Y., Wu, Z.Y., Chen, H.L., Application status of biocides and fungicide in shoe material and its determination methods, China Leather, 2010, 10, 110-115.

6. Corning, D.R., Cory, N.J., Sykes, R.L., Studies on pesticides used for the protection of skins during storage and transportation. Part 2: Analytical studies and environmental impact, J Soc Leath Tech Ch, 1993, 77, 3-13.

7. Golob, P., Robinson, F.V., Gutu, S., Studies on pesticides used for the protection of skins during storage and transportation. Part 1: Protection of goat skins against beetle damage using insecticides in Zimbabwe, I Soc Leath Tech Ch, 1992, 76, 83-88.

8. Jin, J., Yu, L.Y., Dong, W., Wu, M.R., Liu, S.R., Study on determination of carbendazim, chlorothalonil and thiophanate-methyl in Leather, Leath Ch, 2014, 31, 16-20.

9. Li, Z.J., Yang, W.H., Qiu, M.J., Guangdong chemical industry, 2002, 2, 17-19.

10. Nunes, R.R., Bontempi, R.M., Mendonça, G., Galetti, G., Rezende, M.O.O., Vermicomposting as an advanced biological treatment for industrial waste from the leather industry, $J$ Environ Sci Health B, 2016, 51, 5, 271-277.

11. De La Cruz, A.R.H., Ferreira, L.D.S.C., Andrade, V.P., Gioda, A., Biomonitoring of Toxic Elements in Plants Collected Near Leather Tanning Industry, J Braz Chem Soc, 2019, 30, 2, 256-264, https://doi.org/10.21577/01035053.20180174.

12. Shi, J.J., Chen, H.L., Arocena, J.M., Whitcombe, T., Thrig, R.W., Memiaghe, J.N., Elemental sulfur amendment decreases bio-available $\mathrm{Cr}-\mathrm{VI}$ in soils impacted by leather tanneries, Environ Pollut, 2016, 212, 57-64, https://doi. org/10.1016/j.envpol.2016.01.045.

13. Balaji, A.P.B., Sastry, T.P., Manigandan, S., Mukherjee, A., Chandrasekara, N., Environmental benignity of a pesticide in soft colloidal hydrodispersive nanometric form with improved toxic precision towards the target organisms than non-target organisms, Sci Total Environ, 2017, 579, 190-201, https:// doi.org/10.1016/j.scitotenv.2016.10.240.

14. Sundaramoorthy, R., Velusamy, Y., Balaji, A.P.B., Mukherjee, A., Chandrasekaran, N., Comparative cytotoxic and genotoxic effects of permethrin and its nanometric form on human erythrocytes and lymphocytes in vitro, Chem Biol Interact, 2016, 257, 119-124, https://doi.org/10.1016/j.cbi.2016.08.001.

15. Fritschi, L., Benke, G., Risch, H.A., Schulte, A., Webb, P.M., Whiteman, D.C., Fawcett, J., Neale, R.E., Occupational exposure to $\mathrm{N}$-nitrosamines and pesticides and risk of pancreatic cancer, Occup Environ Med, 2015, 72, 9, 678-683, https://doi.org/10.1136/ oemed-2014-102522.

16. Mikoczy, Z., Hagmar, L., Cancer incidence in the Swedish leather tanning industry: updated findings 1958-99, Occup Environ Med, 2005, 62, 7, 461-464, https://doi. org/10.1136/oem.2004.017038.

17. Heudorf, U., Angerer, J., Drexler, H., Current internal exposure to pesticides in children and adolescents in Germany: Blood plasma levels of pentachlorophenol (PCP), lindane $(\gamma-\mathrm{HCH})$, and dichloro(diphenyl)ethylene (DDE), a biostable metabolite of dichloro(diphenyl) trichloroethane (DDT), Int J Hyg Environ Health, 2003, 206, 6, 485-491.

18. Heudorf, U., Angerer, J., Drexler, H., Current internal exposure to pesticides in children and adolescents in Germany: urinary levels of metabolites of pyrethroid and organophosphorus insecticides, Int Arch Occup Environ Health, 2004, 77, 67-72, 
https://doi.org/10.1007/s00420-003-0470-5.

19. ISO/TR 16178:2012 Footwear Critical substances potentially present in footwear and footwear components.

20. GB/T 18412.1-2006 The detection of pesticide residues in textiles Part one: 77 kinds of pesticides.

21.GB/T 18412.2-2006 The detection of pesticide residues in textiles Part two: organochlorine pesticides.

22. GB/T 23204-2008 The detection of 519 kinds of pesticide residues and related chemicals in Chinese tea by GC-MS.

23. Gong, T.S., Dong, S.M., Lin, F., Lian, X.B., Determination of Salicylanilide in Leather Fungicides by HPLC, Leather Science and Industry, 2011, 21, 43-47.

24. Frenich, A.G., Romero-González, R., Gómez-Pérez, M.L., Martinez Vidal, J.L., Multi-mycotoxin analysis in eggs using a QuEChERS-based extraction procedure and ultra-high-pressure liquid chromatography coupled to triple quadrupole mass spectrometry, J Chromatogr A, 2011, 1218, 28, 4349-4356, https://doi.org/10.1016/j. chroma.2011.05.005.

25. Xian, Y., Dong, H., Wu, Y., Guo, X., Hou, X., Wang, B., QuEChERS-based purification method coupled to ultrahigh performance liquid chromatography-tandem mass spectrometry (UPLC-MS/MS) to determine six quaternary ammonium compounds (QACs) in dairy products, Food Chem, 2016, 212, 96-103, https://doi.org/10.1016/j. foodchem.2016.05.151.

26. Xian, Y., Guo, X., Hou, X., Wang, L., Wu, Y., Chen, L., Dong, H., Wang, B., A modified quick, easy, cheap, effective, rugged, and safe cleanup method followed by liquid chromatography-tandem mass spectrometry for the rapid analysis of perchlorate, bromate and hypophosphite in flour, $J$ Chromatogr $A$, 2017, 1526(Supplement C), 31-38, https:// doi.org/10.1016/j.chroma.2017.10.047.

27. Xian Y., Wu, Y., Dong, H., Guo, X., Wang, B., Wang, L., Dispersive micro solid phase extraction (DMSPE) using polymer anion exchange (PAX) as the sorbent followed by UPLC-MS/MS for the rapid determination of four bisphenols in commercial edible oils, J Chromatogr A, 2017, 1517(Supplement C), 35-43, https://doi.org/10.1016/j. chroma.2017.08.067.

(C) 2020 by the author(s). Published by INCDTPICPI, Bucharest, RO. This is an open access article distributed under the terms and conditions of the Creative Commons Attribution license (http:// creativecommons.org/licenses/by/4.0/). 


\section{SUPPLEMENTAL MATERIALS}

Table S1: Detailed information of 24 pesticides

\begin{tabular}{|c|c|c|c|}
\hline Series Number & Pesticide & CAS number & Chemical formula \\
\hline 18 & $0, p^{\prime}-D D T$ & $789-02-6$ & $\mathrm{C}_{14} \mathrm{H}_{9} \mathrm{Cl}_{5}$ \\
\hline 21 & $p, p^{\prime}-D D T$ & $50-29-3$ & $\mathrm{C}_{14} \mathrm{H}_{9} \mathrm{Cl}_{5}$ \\
\hline 17 & $o, p^{\prime}-D D D$ & $53-19-0$ & $\mathrm{C}_{14} \mathrm{H}_{10} \mathrm{Cl}_{4}$ \\
\hline 19 & $p, p^{\prime}-D D D$ & $72-54-8$ & $\mathrm{C}_{14} \mathrm{H}_{8} \mathrm{Cl}_{4}$ \\
\hline 12 & $o, p^{\prime}-D D E$ & $3424-82-6$ & $\mathrm{C}_{14} \mathrm{H}_{8} \mathrm{Cl}_{4}$ \\
\hline 15 & $p, p^{\prime}-D D E$ & $72-55-9$ & $\mathrm{C}_{14} \mathrm{H}_{8} \mathrm{Cl}_{4}$ \\
\hline 2 & $\alpha-B H C$ & $319-84-6$ & $\mathrm{C}_{6} \mathrm{H}_{6} \mathrm{Cl}_{6}$ \\
\hline 6 & $\beta-\mathrm{BHC}$ & $319-85-7$ & $\mathrm{C}_{6} \mathrm{H}_{6} \mathrm{Cl}_{6}$ \\
\hline 7 & $\delta-\mathrm{BHC}$ & $319-86-8$ & $\mathrm{C}_{6} \mathrm{H}_{6} \mathrm{Cl}_{6}$ \\
\hline 3 & Lindane & $58-89-9$ & $\mathrm{C}_{6} \mathrm{H}_{6} \mathrm{Cl}_{6}$ \\
\hline 8 & Malathion & $121-75-5$ & $\mathrm{C}_{10} \mathrm{H}_{19} \mathrm{O}_{6} \mathrm{PS}_{2}$ \\
\hline 23 & Methoxychlor & $72-43-5$ & $\mathrm{C}_{16} \mathrm{H}_{15} \mathrm{Cl}_{3} \mathrm{O}_{2}$ \\
\hline 4 & Aldrin & $309-00-2$ & $\mathrm{C}_{12} \mathrm{H}_{8} \mathrm{Cl}_{6}$ \\
\hline 16 & Dieldrin & $60-57-1$ & $\mathrm{C}_{12} \mathrm{H}_{8} \mathrm{Cl}_{6} \mathrm{O}$ \\
\hline 10 & Ethylparathion & $56-38-2$ & $\mathrm{C}_{10} \mathrm{H}_{14} \mathrm{NO}_{5} \mathrm{PS}$ \\
\hline 13 & $\alpha$-Endosulfan & $115-29-7$ & $\mathrm{C}_{9} \mathrm{H}_{6} \mathrm{Cl}_{6} \mathrm{O}_{3} \mathrm{~S}$ \\
\hline 20 & $\beta$-Endosulfan & $33213-65-9$ & $\mathrm{C}_{9} \mathrm{H}_{6} \mathrm{Cl}_{6} \mathrm{O}_{3} \mathrm{~S}$ \\
\hline 22 & Mirex & $2385-85-5$ & $\mathrm{C}_{10} \mathrm{Cl}_{12}$ \\
\hline 9 & Dichlofluanide & 1085-98-9 & $\mathrm{C}_{9} \mathrm{H}_{11} \mathrm{Cl}_{2} \mathrm{FN}_{2} \mathrm{O}_{2} \mathrm{~S}_{2}$ \\
\hline 11 & Heptachloroepoxide & $1024-57-3$ & $\mathrm{C}_{10} \mathrm{H}_{5} \mathrm{Cl}_{7} \mathrm{O}$ \\
\hline 1 & Pentachloroanisole & $1825-21-4$ & $\mathrm{C}_{7} \mathrm{H}_{3} \mathrm{Cl}_{5} \mathrm{O}$ \\
\hline 24 & Permethrin & $52645-53-1$ & $\mathrm{C}_{21} \mathrm{H}_{20} \mathrm{Cl}_{2} \mathrm{O}_{3}$ \\
\hline 14 & Tolyfluanide & $731-27-1$ & $\mathrm{C}_{10} \mathrm{H}_{13} \mathrm{Cl}_{2} \mathrm{FN}_{2} \mathrm{O}_{2} \mathrm{~S}_{2}$ \\
\hline 5 & Chlorothalonil & $1897-45-6$ & $\mathrm{C}_{8} \mathrm{Cl}_{4} \mathrm{~N}_{2}$ \\
\hline
\end{tabular}

Table S2: The extraction effect of different ratio of $n$-hexane-ethyl acetate on 24 pesticide residues in leather sample

\begin{tabular}{ccccccccc}
\hline Analyte & $\begin{array}{c}\text { Concentration } \\
\text { (added) } \\
\mathrm{ng} \mathrm{mL}^{-1}\end{array}$ & $5: 1$ & $3: 1$ & $2: 1$ & $1: 1$ & $1: 2$ & $1: 3$ & $1: 5$ \\
\hline 1 & 200 & 104.99 & 98.51 & 88.60 & 93.24 & 81.09 & 82.34 & 78.46 \\
2 & 200 & 100.78 & 97.57 & 84.05 & 101.75 & 85.72 & 87.86 & 84.93 \\
3 & 200 & 96.56 & 87.19 & 76.52 & 110.41 & 92.88 & 92.70 & 92.99 \\
4 & 200 & 94.36 & 88.54 & 85.45 & 105.98 & 87.90 & 93.11 & 91.09 \\
5 & 200 & 107.82 & 42.40 & 55.44 & 57.66 & 45.33 & 53.45 & 39.20 \\
6 & 200 & 94.44 & 89.55 & 92.92 & 99.81 & 83.66 & 86.03 & 85.45 \\
7 & 200 & 129.78 & 119.05 & 111.83 & 117.92 & 104.36 & 109.34 & 104.68 \\
8 & 200 & 132.41 & 121.99 & 114.86 & 127.30 & 115.29 & 118.32 & 116.72 \\
9 & 200 & 53.49 & 53.14 & 55.72 & 53.94 & 36.75 & 23.64 & 27.11 \\
10 & 200 & 142.86 & 131.11 & 124.11 & 126.81 & 112.13 & 114.13 & 118.79 \\
11 & 200 & 92.14 & 85.07 & 79.99 & 96.68 & 86.44 & 92.69 & 88.78 \\
12 & 200 & 91.31 & 85.71 & 86.32 & 103.70 & 84.83 & 94.50 & 89.10 \\
13 & 200 & 138.75 & 162.17 & 130.85 & 112.79 & 149.26 & 147.74 & 126.97 \\
\hline
\end{tabular}




\begin{tabular}{lcccccccc}
\hline 14 & 200 & 95.41 & 57.25 & 46.34 & 57.29 & 44.17 & 29.61 & 31.04 \\
15 & 200 & 97.04 & 87.37 & 83.97 & 99.91 & 85.19 & 91.74 & 89.24 \\
16 & 200 & 98.00 & 84.55 & 81.43 & 109.29 & 86.50 & 85.50 & 83.50 \\
17 & 200 & 113.23 & 106.95 & 100.65 & 104.25 & 93.18 & 97.53 & 93.69 \\
18 & 200 & 55.05 & 42.23 & 37.00 & 120.70 & 91.48 & 98.33 & 105.27 \\
19 & 200 & 124.64 & 113.29 & 105.25 & 111.34 & 101.00 & 104.92 & 97.56 \\
20 & 200 & 142.04 & 141.02 & 129.22 & 109.96 & 105.81 & 89.64 & 100.15 \\
21 & 200 & 37.85 & 23.78 & 20.62 & 114.72 & 83.86 & 92.14 & 106.92 \\
22 & 200 & 77.31 & 67.89 & 55.64 & 93.00 & 77.19 & 83.76 & 83.86 \\
23 & 200 & 44.29 & 29.60 & 27.31 & 110.22 & 84.68 & 94.79 & 101.00 \\
24 & 200 & 101.22 & 136.02 & 117.00 & 107.80 & 94.05 & 119.83 & 129.82 \\
\hline
\end{tabular}

Table S2A: Recovery and precision test in Cowhide sample

\begin{tabular}{|c|c|c|c|c|c|}
\hline Analyte & $\begin{array}{l}\text { Added } \\
(\mu \mathrm{g} / \mathrm{kg})\end{array}$ & $\begin{array}{c}\text { Found } \\
\text { Average } \\
(\mathrm{n}=6, \mu \mathrm{g} / \mathrm{kg})\end{array}$ & $\begin{array}{c}\text { Average } \\
\text { recovery } \\
(\%) \\
\end{array}$ & $\mathrm{SD}(\mu \mathrm{g} / \mathrm{kg})$ & $\begin{array}{l}\text { RSD } \\
(\%)\end{array}$ \\
\hline \multirow{3}{*}{1} & 100 & 107.1 & 107.1 & 2.41 & 2.25 \\
\hline & 300 & 322.6 & 107.5 & 8.61 & 2.66 \\
\hline & 600 & 666.6 & 111.1 & 22.3 & 3.35 \\
\hline \multirow{3}{*}{2} & 100 & 109.9 & 109.9 & 5.98 & 5.44 \\
\hline & 300 & 331.8 & 110.6 & 8.62 & 2.61 \\
\hline & 600 & 676.3 & 112.7 & 20.4 & 3.01 \\
\hline \multirow{3}{*}{3} & 100 & 80.0 & 80.0 & 4.20 & 5.25 \\
\hline & 300 & 303.1 & 101.0 & 16.9 & 5.58 \\
\hline & 600 & 636.4 & 106.1 & 21.6 & 3.40 \\
\hline \multirow{3}{*}{4} & 100 & 113.3 & 113.3 & 7.21 & 6.37 \\
\hline & 300 & 337.7 & 112.6 & 9.61 & 2.84 \\
\hline & 600 & 700.9 & 116.8 & 19.1 & 2.73 \\
\hline \multirow{3}{*}{5} & 100 & 78.4 & 78.4 & 5.68 & 7.24 \\
\hline & 300 & 312.3 & 104.1 & 34.8 & 11.16 \\
\hline & 600 & 733.0 & 122.2 & 62.3 & 8.50 \\
\hline \multirow{3}{*}{6} & 100 & 83.6 & 83.6 & 3.87 & 4.63 \\
\hline & 300 & 306.7 & 102.2 & 9.00 & 2.93 \\
\hline & 600 & 660.2 & 110.0 & 24.9 & 3.77 \\
\hline \multirow{3}{*}{7} & 100 & 91.8 & 91.8 & 6.05 & 6.60 \\
\hline & 300 & 336.1 & 112.0 & 10.2 & 3.02 \\
\hline & 600 & 681.6 & 113.6 & 20.8 & 3.05 \\
\hline \multirow{3}{*}{8} & 100 & 114.9 & 114.9 & 10.8 & 9.40 \\
\hline & 300 & 394.8 & 131.6 & 35.2 & 8.90 \\
\hline & 600 & 675.8 & 112.6 & 24.2 & 3.59 \\
\hline \multirow{3}{*}{9} & 100 & 114.3 & 114.3 & 6.09 & 5.33 \\
\hline & 300 & 330.7 & 110.2 & 28.4 & 8.60 \\
\hline & 600 & 732.4 & 122.1 & 14.9 & 2.03 \\
\hline \multirow{3}{*}{10} & 100 & 114.4 & 114.4 & 9.36 & 8.18 \\
\hline & 300 & 335.0 & 111.7 & 6.2 & 1.84 \\
\hline & 600 & 702.3 & 117.0 & 13.4 & 1.91 \\
\hline \multirow{3}{*}{11} & 100 & 117.1 & 117.1 & 4.26 & 3.64 \\
\hline & 300 & 343.1 & 114.4 & 7.41 & 2.16 \\
\hline & 600 & 709.2 & 118.2 & 20.9 & 2.95 \\
\hline \multirow{3}{*}{12} & 100 & 109.0 & 109.0 & 4.76 & 4.37 \\
\hline & 300 & 337.4 & 112.5 & 11.0 & 3.27 \\
\hline & 600 & 693.7 & 115.6 & 21.8 & 3.14 \\
\hline \multirow{3}{*}{13} & 100 & 115.2 & 115.2 & 3.85 & 3.34 \\
\hline & 300 & 394.0 & 131.3 & 22.3 & 5.67 \\
\hline & 600 & 762.2 & 127.0 & 33.8 & 4.43 \\
\hline
\end{tabular}




\begin{tabular}{|c|c|c|c|c|c|}
\hline Analyte & $\begin{array}{l}\text { Added } \\
(\mu \mathrm{g} / \mathrm{kg})\end{array}$ & $\begin{array}{c}\text { Found } \\
\text { Average } \\
(n=6, \mu g / k g)\end{array}$ & $\begin{array}{c}\text { Average } \\
\text { recovery } \\
(\%)\end{array}$ & $\mathrm{SD}(\mu \mathrm{g} / \mathrm{kg})$ & $\begin{array}{l}\text { RSD } \\
\text { (\%) }\end{array}$ \\
\hline & 100 & 110.2 & 110.2 & 10.1 & 9.14 \\
\hline \multirow[t]{3}{*}{14} & 300 & 341.9 & 114.0 & 39.2 & 11.46 \\
\hline & 600 & 574.5 & 95.8 & 19.6 & 3.41 \\
\hline & 100 & 118.2 & 118.2 & 3.91 & 3.31 \\
\hline \multirow[t]{3}{*}{15} & 300 & 340.2 & 113.4 & 9.72 & 2.85 \\
\hline & 600 & 695.5 & 115.9 & 23.0 & 3.31 \\
\hline & 100 & 90.6 & 90.6 & 7.80 & 8.60 \\
\hline \multirow[t]{3}{*}{16} & 300 & 217.1 & 72.4 & 24.3 & 11.18 \\
\hline & 600 & 482.1 & 80.3 & 37.9 & 7.85 \\
\hline & 100 & 127.5 & 127.5 & 6.49 & 5.09 \\
\hline \multirow[t]{3}{*}{17} & 300 & 392.8 & 130.9 & 21.7 & 5.52 \\
\hline & 600 & 737.0 & 122.8 & 45.3 & 6.15 \\
\hline & 100 & 108.7 & 108.7 & 3.95 & 3.63 \\
\hline \multirow[t]{3}{*}{18} & 300 & 323.9 & 108.0 & 15.3 & 4.72 \\
\hline & 600 & 653.1 & 108.8 & 24.9 & 3.81 \\
\hline & 100 & 113.5 & 113.5 & 8.84 & 7.79 \\
\hline \multirow[t]{3}{*}{19} & 300 & 368.7 & 122.9 & 25.73 & 6.98 \\
\hline & 600 & 680.2 & 113.4 & 49.4 & 7.26 \\
\hline & 100 & 95.2 & 95.2 & 6.72 & 7.05 \\
\hline \multirow[t]{3}{*}{20} & 300 & 290.2 & 96.7 & 32.3 & 11.14 \\
\hline & 600 & 552.6 & 92.1 & 51.3 & 9.29 \\
\hline & 100 & 106.0 & 106.0 & 9.11 & 8.59 \\
\hline \multirow[t]{3}{*}{21} & 300 & 314.8 & 104.9 & 27.5 & 8.75 \\
\hline & 600 & 616.3 & 102.7 & 52.3 & 8.48 \\
\hline & 100 & 104.6 & 104.6 & 2.37 & 2.27 \\
\hline \multirow[t]{3}{*}{22} & 300 & 318.4 & 106.1 & 7.1 & 2.23 \\
\hline & 600 & 655.9 & 109.3 & 17.2 & 2.63 \\
\hline & 100 & 102.5 & 102.5 & 9.59 & 9.36 \\
\hline \multirow[t]{3}{*}{23} & 300 & 325.1 & 108.4 & 12.9 & 3.98 \\
\hline & 600 & 645.0 & 107.5 & 26.1 & 4.04 \\
\hline & 100 & 124.8 & 124.8 & 8.35 & 6.69 \\
\hline \multirow[t]{2}{*}{24} & 300 & 373.9 & 124.6 & 12.8 & 3.41 \\
\hline & 600 & 763.7 & 127.3 & 25.7 & 3.37 \\
\hline
\end{tabular}

Table S2B: Recovery and precision test in sheep leather sample $(n=6)$

\begin{tabular}{|c|c|c|c|c|c|}
\hline Analyte & $\begin{array}{l}\text { Added } \\
(\mu \mathrm{g} / \mathrm{kg})\end{array}$ & $\begin{array}{c}\text { Found } \\
\text { Average } \\
(\mathrm{n}=6, \mu \mathrm{g} / \mathrm{kg})\end{array}$ & $\begin{array}{c}\text { Average } \\
\text { recovery } \\
(\%)\end{array}$ & $\mathrm{SD}(\mu \mathrm{g} / \mathrm{kg})$ & $\begin{array}{l}\text { RSD } \\
\text { (\%) }\end{array}$ \\
\hline \multirow{4}{*}{1} & 100 & 107.97 & 107.97 & 1.42 & 1.32 \\
\hline & 300 & 325.42 & 108.47 & 4.77 & 1.46 \\
\hline & 600 & 667.72 & 111.29 & 10.76 & 1.61 \\
\hline & 100 & 100.90 & 100.90 & 3.06 & 3.03 \\
\hline \multirow[t]{3}{*}{2} & 300 & 326.18 & 108.73 & 4.58 & 1.40 \\
\hline & 600 & 676.63 & 112.77 & 13.22 & 1.95 \\
\hline & 100 & 105.94 & 105.94 & 3.58 & 3.38 \\
\hline \multirow[t]{3}{*}{3} & 300 & 323.86 & 107.95 & 5.70 & 1.76 \\
\hline & 600 & 667.29 & 111.22 & 12.32 & 1.85 \\
\hline & 100 & 114.16 & 114.16 & 2.63 & 2.30 \\
\hline \multirow[t]{3}{*}{4} & 300 & 342.56 & 114.19 & 5.11 & 1.49 \\
\hline & 600 & 700.92 & 116.82 & 11.80 & 1.68 \\
\hline & 100 & 78.36 & 78.36 & 1.97 & 2.52 \\
\hline \multirow[t]{2}{*}{5} & 300 & 231.27 & 77.09 & 11.65 & 5.04 \\
\hline & 600 & 492.19 & 82.03 & 44.17 & 8.97 \\
\hline
\end{tabular}




\begin{tabular}{|c|c|c|c|c|c|}
\hline Analyte & $\begin{array}{l}\text { Added } \\
(\mu \mathrm{g} / \mathrm{kg})\end{array}$ & $\begin{array}{c}\text { Found } \\
\text { Average } \\
(n=6, \mu g / k g)\end{array}$ & $\begin{array}{l}\text { Average } \\
\text { recovery } \\
(\%)\end{array}$ & $\mathrm{SD}(\mu \mathrm{g} / \mathrm{kg})$ & $\begin{array}{l}\text { RSD } \\
(\%)\end{array}$ \\
\hline \multirow{3}{*}{6} & 100 & 107.61 & 107.61 & 2.43 & 2.26 \\
\hline & 300 & 333.12 & 111.04 & 6.36 & 1.91 \\
\hline & 600 & 687.42 & 114.57 & 16.79 & 2.44 \\
\hline \multirow{3}{*}{7} & 100 & 106.23 & 106.23 & 5.94 & 5.59 \\
\hline & 300 & 311.20 & 103.73 & 6.50 & 2.09 \\
\hline & 600 & 640.76 & 106.79 & 16.96 & 2.65 \\
\hline \multirow{3}{*}{8} & 100 & 111.49 & 111.49 & 3.77 & 3.39 \\
\hline & 300 & 387.82 & 129.27 & 5.97 & 1.54 \\
\hline & 600 & 746.45 & 124.41 & 16.27 & 2.18 \\
\hline \multirow{3}{*}{9} & 100 & 72.78 & 72.78 & 5.70 & 7.84 \\
\hline & 300 & 240.82 & 80.27 & 13.07 & 5.43 \\
\hline & 600 & 480.29 & 80.05 & 14.65 & 3.05 \\
\hline \multirow{3}{*}{10} & 100 & 87.98 & 87.98 & 3.90 & 4.43 \\
\hline & 300 & 363.85 & 121.28 & 8.10 & 2.23 \\
\hline & 600 & 649.37 & 108.23 & 14.74 & 2.27 \\
\hline \multirow{3}{*}{11} & 100 & 118.06 & 118.06 & 4.40 & 3.72 \\
\hline & 300 & 348.46 & 116.15 & 7.72 & 2.21 \\
\hline & 600 & 653.41 & 108.90 & 14.48 & 2.22 \\
\hline \multirow{3}{*}{12} & 100 & 118.33 & 118.33 & 3.38 & 2.86 \\
\hline & 300 & 351.02 & 117.01 & 5.77 & 1.64 \\
\hline & 600 & 653.99 & 109.00 & 13.68 & 2.09 \\
\hline \multirow{3}{*}{13} & 100 & 103.37 & 103.37 & 6.18 & 5.98 \\
\hline & 300 & 377.60 & 125.87 & 20.07 & 5.32 \\
\hline & 600 & 679.32 & 113.22 & 12.90 & 1.90 \\
\hline \multirow{3}{*}{14} & 100 & 81.10 & 81.10 & 7.51 & 9.26 \\
\hline & 300 & 262.55 & 87.52 & 25.76 & 9.81 \\
\hline & 600 & 528.26 & 88.04 & 16.33 & 3.09 \\
\hline \multirow{3}{*}{15} & 100 & 118.80 & 118.80 & 2.42 & 2.03 \\
\hline & 300 & 358.31 & 119.44 & 6.69 & 1.87 \\
\hline & 600 & 664.75 & 110.79 & 15.21 & 2.29 \\
\hline \multirow{3}{*}{16} & 100 & 103.82 & 103.82 & 3.16 & 3.04 \\
\hline & 300 & 329.67 & 109.89 & 13.56 & 4.11 \\
\hline & 600 & 659.88 & 109.98 & 16.17 & 2.45 \\
\hline \multirow{3}{*}{17} & 100 & 104.65 & 104.65 & 7.96 & 7.60 \\
\hline & 300 & 380.88 & 126.96 & 22.95 & 6.03 \\
\hline & 600 & 737.28 & 122.88 & 45.95 & 6.23 \\
\hline \multirow{3}{*}{18} & 100 & 100.54 & 100.54 & 8.60 & 8.56 \\
\hline & 300 & 304.30 & 101.43 & 24.92 & 8.19 \\
\hline & 600 & 560.08 & 93.35 & 41.59 & 7.43 \\
\hline \multirow{3}{*}{19} & 100 & 98.32 & 98.32 & 10.58 & 10.76 \\
\hline & 300 & 395.68 & 131.89 & 30.27 & 7.65 \\
\hline & 600 & 724.84 & 120.81 & 55.60 & 7.67 \\
\hline \multirow{3}{*}{20} & 100 & 79.73 & 79.73 & 6.22 & 7.80 \\
\hline & 300 & 304.06 & 101.35 & 15.80 & 5.20 \\
\hline & 600 & 617.20 & 102.87 & 15.47 & 2.51 \\
\hline \multirow{3}{*}{21} & 100 & 81.23 & 81.23 & 2.57 & 3.16 \\
\hline & 300 & 318.60 & 106.20 & 6.92 & 2.17 \\
\hline & 600 & 561.11 & 93.52 & 26.22 & 4.67 \\
\hline \multirow{3}{*}{22} & 100 & 109.71 & 109.71 & 4.83 & 4.40 \\
\hline & 300 & 327.41 & 109.14 & 4.18 & 1.28 \\
\hline & 600 & 604.07 & 100.68 & 7.49 & 1.24 \\
\hline
\end{tabular}




\begin{tabular}{cccccc}
\hline Analyte & $\begin{array}{c}\text { Added } \\
(\mu \mathrm{g} / \mathrm{kg})\end{array}$ & $\begin{array}{c}\text { Found } \\
\text { Average } \\
(\mathrm{n}=6, \mu \mathrm{g} / \mathrm{kg})\end{array}$ & $\begin{array}{c}\text { Average } \\
\text { recovery } \\
(\%)\end{array}$ & SD $(\mu \mathrm{g} / \mathrm{kg})$ & $\begin{array}{c}\text { RSD } \\
(\%)\end{array}$ \\
\hline \multirow{2}{*}{23} & 100 & 93.40 & 93.40 & 9.56 & 10.23 \\
& 300 & 280.94 & 93.65 & 27.78 & 9.89 \\
& 600 & 511.95 & 85.33 & 46.06 & 9.00 \\
24 & 100 & 107.68 & 107.68 & 4.05 & 3.76 \\
& 300 & 364.06 & 121.35 & 8.80 & 2.42 \\
& 600 & 657.94 & 109.66 & 17.62 & 2.68 \\
\hline
\end{tabular}

Table S2C: Recovery and precision test in pig leather sample $(n=6)$

\begin{tabular}{|c|c|c|c|c|c|}
\hline Analyte & $\begin{array}{l}\text { Added } \\
(\mu \mathrm{g} / \mathrm{kg})\end{array}$ & $\begin{array}{l}\text { Average content } \\
\quad(n=6, \mu \mathrm{g} / \mathrm{kg})\end{array}$ & $\begin{array}{c}\text { Average } \\
\text { recovery } \\
(\%)\end{array}$ & $\mathrm{SD}(\mu \mathrm{g} / \mathrm{kg})$ & $\begin{array}{l}\text { RSD } \\
(\%)\end{array}$ \\
\hline \multirow{3}{*}{1} & 100 & 80.53 & 80.5 & 5.96 & 7.40 \\
\hline & 300 & 228.53 & 76.18 & 2.93 & 1.28 \\
\hline & 600 & 509.46 & 84.91 & 9.88 & 1.94 \\
\hline \multirow{3}{*}{2} & 100 & 111.32 & 111.3 & 9.66 & 8.68 \\
\hline & 300 & 239.73 & 79.91 & 9.69 & 4.04 \\
\hline & 600 & 535.16 & 89.19 & 9.28 & 1.73 \\
\hline \multirow{3}{*}{3} & 100 & 104.72 & 104.7 & 7.33 & 7.00 \\
\hline & 300 & 291.07 & 97.02 & 26.79 & 9.21 \\
\hline & 600 & 585.58 & 97.60 & 12.26 & 2.09 \\
\hline \multirow{3}{*}{4} & 100 & 110.65 & 110.65 & 9.92 & 8.97 \\
\hline & 300 & 282.99 & 94.33 & 5.86 & 2.07 \\
\hline & 600 & 602.71 & 100.45 & 9.53 & 1.58 \\
\hline \multirow{3}{*}{5} & 100 & 82.92 & 82.92 & 8.57 & 10.33 \\
\hline & 300 & 347.82 & 115.94 & 19.41 & 5.58 \\
\hline & 600 & 578.61 & 96.44 & 15.99 & 2.76 \\
\hline \multirow{3}{*}{6} & 100 & 100.20 & 100.2 & 9.12 & 9.10 \\
\hline & 300 & 335.92 & 111.97 & 12.80 & 3.81 \\
\hline & 600 & 542.62 & 90.44 & 49.73 & 9.17 \\
\hline \multirow{3}{*}{7} & 100 & 116.45 & 116.5 & 11.98 & 10.29 \\
\hline & 300 & 331.24 & 110.41 & 8.46 & 2.55 \\
\hline & 600 & 573.56 & 95.59 & 15.60 & 2.72 \\
\hline \multirow{3}{*}{8} & 100 & 110.17 & 110.2 & 11.47 & 10.41 \\
\hline & 300 & 330.24 & 110.08 & 20.94 & 6.34 \\
\hline & 600 & 682.62 & 113.77 & 56.30 & 8.25 \\
\hline \multirow{3}{*}{9} & 100 & 90.58 & 90.58 & 9.65 & 10.66 \\
\hline & 300 & 270.29 & 90.10 & 36.70 & 13.58 \\
\hline & 600 & 545.94 & 90.99 & 14.30 & 2.62 \\
\hline \multirow{3}{*}{10} & 100 & 123.00 & 123.00 & 12.09 & 9.83 \\
\hline & 300 & 298.87 & 99.62 & 7.88 & 2.64 \\
\hline & 600 & 607.05 & 101.17 & 11.69 & 1.93 \\
\hline \multirow{3}{*}{11} & 100 & 119.42 & 119.42 & 6.61 & 5.54 \\
\hline & 300 & 289.43 & 96.48 & 6.44 & 2.23 \\
\hline & 600 & 604.65 & 100.77 & 9.24 & 1.53 \\
\hline \multirow{3}{*}{12} & 100 & 99.87 & 99.87 & 4.34 & 4.34 \\
\hline & 300 & 280.76 & 93.59 & 10.43 & 3.72 \\
\hline & 600 & 608.38 & 101.40 & 8.64 & 1.42 \\
\hline \multirow{3}{*}{13} & 100 & 82.89 & 82.89 & 11.91 & 14.37 \\
\hline & 300 & 381.80 & 127.27 & 12.92 & 3.38 \\
\hline & 600 & 618.21 & 103.04 & 24.07 & 3.89 \\
\hline \multirow{3}{*}{14} & 100 & 92.92 & 92.92 & 12.09 & 13.01 \\
\hline & 300 & 280.07 & 93.36 & 18.58 & 6.63 \\
\hline & 600 & 565.14 & 94.19 & 16.42 & 2.91 \\
\hline
\end{tabular}


Jinlan DAI, Honglei YIN, Hang WEI, Lei ZHOU, Minghua LIU

\begin{tabular}{|c|c|c|c|c|c|}
\hline Analyte & $\begin{array}{l}\text { Added } \\
(\mu \mathrm{g} / \mathrm{kg})\end{array}$ & $\begin{array}{l}\text { Average content } \\
\quad(n=6, \mu g / k g)\end{array}$ & $\begin{array}{c}\text { Average } \\
\text { recovery } \\
(\%)\end{array}$ & $\mathrm{SD}(\mu \mathrm{g} / \mathrm{kg})$ & $\begin{array}{l}\text { RSD } \\
\text { (\%) }\end{array}$ \\
\hline \multirow{3}{*}{15} & 100 & 118.77 & 118.77 & 4.59 & 3.87 \\
\hline & 300 & 257.68 & 85.89 & 6.89 & 2.67 \\
\hline & 600 & 533.98 & 89.00 & 7.56 & 1.42 \\
\hline \multirow{3}{*}{16} & 100 & 84.36 & 84.36 & 11.88 & 14.08 \\
\hline & 300 & 274.41 & 91.47 & 15.69 & 5.72 \\
\hline & 600 & 603.08 & 100.51 & 29.49 & 4.89 \\
\hline \multirow{3}{*}{17} & 100 & 123.49 & 123.49 & 12.61 & 10.21 \\
\hline & 300 & 335.66 & 111.89 & 4.34 & 1.29 \\
\hline & 600 & 727.10 & 121.18 & 7.91 & 1.09 \\
\hline \multirow{3}{*}{18} & 100 & 104.94 & 104.94 & 7.65 & 7.29 \\
\hline & 300 & 235.10 & 78.37 & 2.80 & 1.19 \\
\hline & 600 & 452.92 & 75.49 & 5.37 & 1.18 \\
\hline \multirow{3}{*}{19} & 100 & 129.02 & 129.02 & 9.60 & 7.44 \\
\hline & 300 & 324.75 & 108.25 & 3.71 & 1.14 \\
\hline & 600 & 713.24 & 118.87 & 8.22 & 1.15 \\
\hline \multirow{3}{*}{20} & 100 & 116.48 & 116.48 & 13.40 & 11.50 \\
\hline & 300 & 312.64 & 104.21 & 32.49 & 10.39 \\
\hline & 600 & 645.48 & 107.58 & 39.55 & 6.13 \\
\hline \multirow{3}{*}{21} & 100 & 89.25 & 89.25 & 7.00 & 7.84 \\
\hline & 300 & 258.12 & 86.04 & 7.66 & 2.97 \\
\hline & 600 & 443.00 & 73.83 & 7.23 & 1.63 \\
\hline \multirow{3}{*}{22} & 100 & 105.16 & 105.16 & 2.66 & 2.53 \\
\hline & 300 & 258.73 & 86.24 & 6.25 & 2.42 \\
\hline & 600 & 541.44 & 90.24 & 8.75 & 1.62 \\
\hline \multirow{3}{*}{23} & 100 & 103.27 & 103.27 & 4.37 & 4.23 \\
\hline & 300 & 222.43 & 74.14 & 3.22 & 1.45 \\
\hline & 600 & 462.89 & 77.15 & 6.64 & 1.43 \\
\hline \multirow{3}{*}{24} & 100 & 125.62 & 125.62 & 4.16 & 3.31 \\
\hline & 300 & 304.41 & 101.47 & 4.31 & 1.42 \\
\hline & 600 & 662.45 & 110.41 & 8.21 & 1.24 \\
\hline
\end{tabular}

